Supplementary Information

\title{
Defect Passivation by Amide-Based Hole Transporting interfacial layer Enhanced Perovskite Grain Growth for Efficient p-i-n Perovskite Solar Cells
}

Shin-Yu Wang ${ }^{\mathrm{a}}$, Chih-Ping Chen, ${ }^{\mathrm{tb}}$ Chung-Lin Chung, ${ }^{\mathrm{b}}$ Chun-Wen Hsu, ${ }^{\mathrm{a}}$ Hsiang-Lin Hsu, ${ }^{\mathrm{b}}$ TingHsuan Wu, ${ }^{a}$ Jia-Ying Zhuang, ${ }^{a}$ Chia-Jui Chang, ${ }^{c}$ Hao Ming Chen ${ }^{c}$ and Yuan Jay Chang a,*

\footnotetext{
${ }^{a}$ Department of Chemistry, Tunghai University, No.1727, Sec.4, Taiwan Boulevard, Xitun District, Taichung 40704, Taiwan

${ }^{b}$ Department of Materials Engineering, Ming Chi University of Technology, 84 Gungjuan Rd., Taishan Dist. New Taipei City 24301, Taiwan

${ }^{c}$ Department of Chemistry, National Taiwan University, Taipei 10617, Taiwan.

${ }^{\ddagger}$ Equal contribution first author
}

Contents

1. Experimental

2. ${ }^{1} \mathrm{H}$ and ${ }^{13} \mathrm{C}$ NMR spectra $9-18$

3. UV-visible spectra and PL spectra

4. Oxidative voltammograms of HTMs

5. Theoretical calculation

6. Space Charge Limited Current Measurements (SCLC)

7. UV, TRPL and PL spectra of PSCs device

8. Contact angle and surface energy

9. XRD spectra

10. Cross-section SEM

11. PSCs device transparency

12. EIS spectra

13. PSC device stability

* Corresponding author. Tel.: +886-4-23590121 ext 32224; fax: +886-4-23590426;

e-mail: jaychang@thu.edu.tw 


\section{Experimental}

General Aspects. All the reagents were used as received from the commercial sources, unless otherwise stated. Dichloromethane (DCM), chloroform, dimethyl sulfoxide (DMSO), orthodichlorobenzene (o-DCB) was dried and distilled over the dehydrating agent $\mathrm{CaH}_{2}$. Toluene is dried and distilled over sodium-ketyl radical system. Methanol was distilled over magnesium by forming the magnesium cake. To perform the reaction, oven-dried glassware were used under nitrogen gas atmosphere. The progress of the reaction was monitored by TLC (Thin Layer Chromatography) analysis using Merck silica-gel $\left(60 \mathrm{~F}_{254}\right)$ precoated plates $(0.25 \mathrm{~mm})$. The compounds were visualized to naked-eye under a UV lamp (366 or $254 \mathrm{~nm}$ ) in a UV chamber or using phosphomolybdic acid (PMA) solution as a stain to detect the compound as a spot. The crude product thus obtained after the reaction was purified by silica-gel (100-200 mesh) column chromatography. A combination of ethyl acetate and hexane was used as the mobile phase. Melting points of the compounds were measured by packing the compound in an open capillary on a melting-point apparatus, and the values were corrected. To record the infrared spectra of the compounds, JASCO FT/IR-4100 spectrometer instrument was used by preparing a dry $\mathrm{KBr}$ pellet, and IR signals are quoted in wavenumbers $\left(\mathrm{cm}^{-1}\right) .{ }^{1} \mathrm{H}(400 \mathrm{MHz})$ and ${ }^{13} \mathrm{C}(100 \mathrm{MHz})$ NMR spectra were recorded in ambient temperature using a Bruker Avance FTNMR $(400 \mathrm{MHz})$ spectrometer in deuterated chloroform $\left(\mathrm{CDCl}_{3}\right)$ with TMS as the internal reference. All the chemical shift values were reported in parts per million $\left(\mathrm{ppm}, \delta\right.$ ). All the reported ${ }^{1} \mathrm{H}$ NMR spectra were calibrated with the residual proton solvent peak $\left(\mathrm{CDCl}_{3}, \delta=7.26 \mathrm{ppm}\right)$ or tetramethylsilane ( $\delta=0.00 \mathrm{ppm})$, and the ${ }^{13} \mathrm{C} \mathrm{NMR}$ spectra were referenced to $\mathrm{CDCl}_{3}, \delta=77.16 \mathrm{ppm}$, respectively. The ${ }^{1} \mathrm{H}$ NMR multiplicities are abbreviated as follows: $s$, singlet; $d$, doublet; $t$, triplet; $m$, multiplet; dd, doublet of doublet; dt, doublet of triplet and the coupling constants $(J)$ values were reported in Hertz $(\mathrm{Hz})$. High-resolution mass spectra (HRMS) were recorded on a Q-TOF Micro micromass spectrometer. UV-visible absorption spectra of compounds in solution were recorded on a AGILENT 8453 diode-array spectrophotometer, and the films were recorded on a JASCO V-650 spectrophotometer. Solvents used for this purpose were of spectroscopic grade, and were purchased commercially. 
The starting materials, such as, 9H-carbazole, 4-bromophenol, 1-bromohexane, bromine, 2(tributylstannyl)thiophene, 2-bromothiophene-5-carbaldehyde, bis(triphenylphosphine)palladium(II) dichloride, 4,4,5,5-Tetramethyl-1,3,2-dioxaborolane, 4-bromobenzaldehyde, Tetrakis(triphenylphosphine)palladium(0), Ethyl 2-cyanoacetate, hexylamine were purchased commercially and used without further purification, unless otherwise stated. All four cabarzole derivatives SY1 SY4 were synthesized according to literature reported procedures and were characterized by ${ }^{1} \mathrm{H},{ }^{13} \mathrm{C}$ NMR, IR spectroscopy techniques as described below.

\section{Thermal analyses}

Thermogravimetric analyses (TGA) were performed on a TGA Q500 V20.10 Build 36 instrument under nitrogen gas atmosphere at a heating rate of $20^{\circ} \mathrm{C} / \mathrm{min}$. Differential Scanning Calorimetry (DSC) studies were performed on a DSC Q200 MDSC instrument under nitrogen gas atmosphere at a heating rate of $10^{\circ} \mathrm{C} / \mathrm{min}$.

\section{Device Fabrication and Measurements}

The ITO-coated glass substrates (sheet resistance: $15 \Omega \mathrm{sq}^{-1}$ ) were cleaned ultrasonically with abstergent aqueous solution, deionized water, acetone, and isopropyl alcohol for $20 \mathrm{~min}$, and then dried under a stream of $\mathrm{N}_{2}$. The substrates were then cleaned with air plasma for $10 \mathrm{~min}$. The PEDOT:PSS (CLEVIOS ${ }^{\text {TM }}$ P VP AI4083) film was deposited from solution using a spin-rate of $4000 \mathrm{rpm}$ (30 s) and an annealing temperature of $120{ }^{\circ} \mathrm{C}(20 \mathrm{~min})$. The HTM layers were prepared from a chlorobenzene $(\mathrm{CB})$ solution $(10 \mathrm{mg} / \mathrm{mL})$ and deposited on top of ITO using a spin-rate of $3000 \mathrm{rpm}$. The optimized thickness of the HTM was $10-15 \mathrm{~nm}$. A NiO $x$ film (ca. $20 \mathrm{~nm}$ ) was prepared by spincoating a solution containing the $\mathrm{NiO}_{x}$ precursor [nickel(II) acetylacetonate $(129 \mathrm{mg})$ dissolved in EtOH $(5 \mathrm{~mL})$ containing $\mathrm{HCl}(38 \mathrm{wt} \%, 50 \mu \mathrm{L})]$. The $\mathrm{NiO}_{x}$-coated substrates were then baked at 320 
${ }^{\circ} \mathrm{C}$ for $45 \mathrm{~min}$ in air. For the bilayer HTL, the HTMs were further spin-coated $(8000 \mathrm{rpm}$, from a 3 $\mathrm{mg} / \mathrm{mL}$ solution in $\mathrm{CB}$ ) on top of $\mathrm{NiO}_{x}$. The $\mathrm{MAPbI}_{3}$ precursor solution was prepared by dissolving 1.2 $\mathrm{M} \mathrm{PbI}_{2}$ and MAI (molar ratio, 1:1) in anhydrous DMF/DMSO (4:1). The solution was stirred at 60 ${ }^{\circ} \mathrm{C}$ for $2 \mathrm{~h}$ in an Ar-filled glove box. The perovskite precursor solutions were then spin-coated on the HTL-coated substrates with a two-step spin rate (step 1: $2000 \mathrm{rpm}$ for $10 \mathrm{~s}$; step 2: $4000 \mathrm{rpm}$ for $20 \mathrm{~s}$ ), and then toluene $(100 \mu \mathrm{L})$ was applied rapidly to the substrates to induce fast crystallization. Finally, the sample was annealed at $100{ }^{\circ} \mathrm{C}$ for $10 \mathrm{~min}$ to complete the transformation to the perovskite. $\mathrm{PC}_{61} \mathrm{BM}\left(20 \mathrm{mg} \mathrm{mL}^{-1}\right.$ in anhydrous $\left.\mathrm{CB}\right)$ was deposited; following the deposition of BCP $\left(2 \mathrm{mg} \mathrm{mL}^{-1}\right.$ in IPA), spin-coating was performed at $6000 \mathrm{rpm}$ for $30 \mathrm{~s}$. Finally, the device was completed through the evaporation of $\mathrm{Ag}$ or $\mathrm{Au}$ contact electrodes $(100 \mathrm{~nm})$ at a vacuum level of $10^{-7} \mathrm{~Pa}$ through a shadow mask. The active area of this electrode was fixed at $0.01 \mathrm{~cm}^{2}$.

The device performance was measured inside a glove box. The current-voltage $(J-V)$ properties of the devices were measured using a computer-controlled Keithley 2400 source measurement unit (SMU) and an Enlitech simulator (AAA Class Solar Simulators) under AM 1.5 illumination (1000 W $\mathrm{m}^{-2}$ ). The illumination intensity was calibrated using a standard Si reference cell and a KG-5 filter. EQEs were measured using an Enlitech QE-R spectral response measurement system to calibrate the current densities of the devices. The morphologies of the perovskites were analyzed through FE-SEM (JEOL JSM 6701F). Grazing-incidence wide-angle X-ray spectroscopy (GIWAXS) was performed using a Philips Panalytical-x' PertPROMRD instrument; the incident beam angle was above the critical angle (ca. $0.5^{\circ}$ ). TRPL spectra were recorded using a time-correlated single-photon counting spectrometer (WELLS-001 FX, DongWoo Optron). The pulse laser had a wavelength of $440 \mathrm{~nm}$ and an average power of $1 \mathrm{~mW}$; it was operated with a duration of excitation of $2 \mu \mathrm{s}$. The SCLC method was used to measure the hole mobility of the HTMs under the optimized conditions. Hole- only devices were fabricated having the device structure ITO/PEDOT:PSS/HTM/MoO$/ / \mathrm{Ag}$. The applied voltage ( $\left.V_{\text {appl }}\right)$ was corrected from the built-in voltage $\left(V_{\mathrm{bi}}\right)$; it was the difference in the work function between the bottom electrode (PEDOT:PSS) and the uppermost electrode $\left(\mathrm{MoO}_{3}\right)$. When sufficient voltage was 
applied to these devices, the transport of holes through the HTM layer was limited by the accumulated space-charge. The equation of the SCLC is described by

$$
\mathbf{J}=\frac{9}{8} \varepsilon_{r} \varepsilon_{0} \mu_{h, e} \frac{V^{2}}{L^{3}}
$$

where $\varepsilon_{\mathrm{r}}$ is the dielectric constant of the material, $\varepsilon_{0}$ is the permittivity of free space $\left(8.85 \times 10^{-12} \mathrm{~F} \mathrm{~m}^{-}\right.$ $\left.{ }^{1}\right), \mu_{\mathrm{h}}$ is the hole mobility, $V$ is the device's applied voltage, and $L$ is the HTM thickness (30 nm).

X-ray absorption near edge spectra (XANES): X-ray absorption spectroscopy including x-ray absorption near edge spectra (XANES) and extended $\mathrm{x}$-ray absorption fine structure (EXAFS) at $\mathrm{Pb}$ L3-edge were collected in total-fluorescence-yield mode at ambient condition in Taiwan BL-12B2 of National Synchrotron Radiation Research Center (NSRRC) at SPring-8, JARSI. The electron storage ring was operated at $8.0 \mathrm{GeV}$ with a constant current of $\sim 100 \mathrm{~mA}$. The spectra were obtained with standard procedures, including pre-edge and post-edge background subtraction, normalization with respect to the edge height.

\section{Experiment data}

9-(4-(hexyloxy)phenyl)-9H-carbazole (1). To a three-necked flask containing a mixture of carbazole (5.00 g, $29.9 \mathrm{mmol})$, copper (1.88 g, $29.9 \mathrm{mmol}), \mathrm{K}_{2} \mathrm{CO}_{3}$ (16.53 g, $\left.119.6 \mathrm{mmol}\right)$, and 1-Bromo-4(hexyloxy)benzene $(15.3 \mathrm{~g}, 59.8 \mathrm{mmol})$ were stirred in nitrobenzene reflux $\left(180^{\circ} \mathrm{C}\right)$ for $72 \mathrm{~h}$. After cooling, the reaction was quenched by adding water. The mixture was extracted with methylene chloride and the organic layer dried over anhydrous $\mathrm{MgSO}_{4}$. Evaporation of the solvent gave the crude, which was purified by silica gel with hexane/methylene chloride (6/1) as eluent. The white solid was isolated in $84.9 \%$ yield $(8.72 \mathrm{~g}, 25.42 \mathrm{mmol}) . \delta_{\mathrm{H}}\left(400 \mathrm{MHz}, \mathrm{CDCl}_{3}\right) 8.16(\mathrm{~d}, 2 \mathrm{H}, J=7.76 \mathrm{~Hz}), 7.45$ $(\mathrm{d}, 2 \mathrm{H}, J=8.76 \mathrm{~Hz}), 7.41(\mathrm{~d}, 2 \mathrm{H}, J=7.20 \mathrm{~Hz}), 7.35(\mathrm{~d}, 2 \mathrm{H}, J=8.08 \mathrm{~Hz}), 7.29(\mathrm{t}, 2 \mathrm{H}, J=7.52 \mathrm{~Hz})$, $7.11(\mathrm{dd}, 2 \mathrm{H}, J=7.02,1.88 \mathrm{~Hz}), 4.07(\mathrm{t}, 2 \mathrm{H}, J=6.56 \mathrm{~Hz}), 1.84-1.91(\mathrm{~m}, 2 \mathrm{H}), 1.51-1.58(\mathrm{~m}, 2 \mathrm{H}), 1.40-$ $1.45(\mathrm{~m}, 4 \mathrm{H}), 0.95-0.99(\mathrm{~m}, 3 \mathrm{H}) . \delta_{\mathrm{C}}\left(100 \mathrm{MHz}, \mathrm{CDCl}_{3}\right)$ 158.4, 141.3, 130.0, 128.4, 125.8, 123.0, 120.2, $119.5,115.5,109.6,68.3,31.5,29.2,25.7,22.6,14.0$. MS (FAB, $70 \mathrm{eV}): \mathrm{m} / \mathrm{z}$ (relative intensity) 343 $\left(\mathrm{M}^{+}, 100\right)$; HRMS calcd for $\mathrm{C}_{24} \mathrm{H}_{25} \mathrm{NO}: 343.1936$, found 343.1937 . 
3,6-dibromo-9-(4-(hexyloxy)phenyl)-9H-carbazole (2). A solution of 1 (5.00 g, $14.6 \mathrm{mmol}$ ) and NBS $(5.19 \mathrm{~g}, 29.1 \mathrm{mmol})$ was stirred in methylene chloride at room temperature. After $36 \mathrm{hr}$, the mixture was quenched by adding water. The mixture was extracted with methylene chloride and the organic layer dried over anhydrous $\mathrm{MgSO}_{4}$. Evaporation of the solvent gave the crude, which was purified by silica gel with hexane/methylene chloride (8/1) as eluent. A white solid was obtained in a $95.9 \%$ yield $(6.98 \mathrm{~g}, 14.0 \mathrm{mmol}) . \delta_{\mathrm{H}}\left(400 \mathrm{MHz}, \mathrm{CDCl}_{3}\right) 8.18(\mathrm{~d}, 2 \mathrm{H}, J=1.84 \mathrm{~Hz}), 7.48$ (dd, 2H, $\left.J=8.72,1.92 \mathrm{~Hz}\right)$, 7.33-7.37 (m, 2H), $7.17(\mathrm{~d}, 2 \mathrm{H}, J=8.72 \mathrm{~Hz}), 7.07-7.11(\mathrm{~m}, 2 \mathrm{H}), 4.05(\mathrm{t}, 2 \mathrm{H}, J=6.52 \mathrm{~Hz}), 1.82-1.89$ $(\mathrm{m}, 2 \mathrm{H}), 1.48-1.56(\mathrm{~m}, 2 \mathrm{H}), 1.34-1.43(\mathrm{~m}, 4 \mathrm{H}), 0.92-0.96(\mathrm{~m}, 3 \mathrm{H}) . \delta_{\mathrm{C}}\left(100 \mathrm{MHz}, \mathrm{CDCl}_{3}\right) 158.9,140.3$, 129.2, 129.0, 128.3, 123.6, 123.1, 115.7, 112.7, 111.4, 68.4, 31.6, 29.2, 25.7, 22.6, 14.0. MS (FAB, 70 $\mathrm{eV}): \mathrm{m} / \mathrm{z}$ (relative intensity) $499\left(\mathrm{M}^{+}, 100\right)$; HRMS calcd for $\mathrm{C}_{24} \mathrm{H}_{23}{ }^{79} \mathrm{Br}_{2} \mathrm{NO}: 499.0146$, found 499.0151 .

9-(4-(hexyloxy)phenyl)-3,6-di(thiophen-2-yl)-9H-carbazole (3). A solution of 2 (4.00 g, $8.02 \mathrm{mmol}$ ), 2-(Tributylstannyl)thiophene $(15.0 \mathrm{~g}, 40.1 \mathrm{mmol})$ and $\mathrm{PdCl}_{2}\left(\mathrm{PPh}_{3}\right)_{2}(0.11 \mathrm{~g}, 0.16 \mathrm{mmol})$ was stirred in dry DMF at $90{ }^{\circ} \mathrm{C}$. After $18 \mathrm{hr}$, the mixture was quenched by adding water. The mixture was extracted with methylene chloride and the organic layer dried over anhydrous $\mathrm{MgSO}_{4}$. Evaporation of the solvent gave the crude, which was purified by silica gel with hexane/methylene chloride (6/1) as eluent. A yellow solid was obtained in a $78.1 \%$ yield $(3.17 \mathrm{~g}, 6.26 \mathrm{mmol}) . \delta \mathrm{H}\left(400 \mathrm{MHz}, \mathrm{CDCl}_{3}\right) 8.39$ $(\mathrm{d}, 2 \mathrm{H}, J=1.64 \mathrm{~Hz}), 7.68(\mathrm{dd}, 2 \mathrm{H}, J=8.96,1.04 \mathrm{~Hz}), 7.42-7.46(\mathrm{~m}, 2 \mathrm{H}), 7.37$ (dd, 2H, $J=3.48,0.96$ $\mathrm{Hz}), 7.32(\mathrm{~d}, 2 \mathrm{H}, J=8.4 \mathrm{~Hz}), 7.28(\mathrm{dd}, 2 \mathrm{H}, J=5.24,0.92 \mathrm{~Hz}), 7.10-7.14(\mathrm{~m}, 4 \mathrm{H}), 4.07$ (t, $2 \mathrm{H}, J=6.56$ $\mathrm{Hz}), 1.83-1.90(\mathrm{~m}, 2 \mathrm{H}), 1.50-1.57(\mathrm{~m}, 2 \mathrm{H}), 1.36-1.44(\mathrm{~m}, 4 \mathrm{H}), 0.94-0.97(\mathrm{~m}, 3 \mathrm{H}) . \delta_{\mathrm{C}}\left(100 \mathrm{MHz}, \mathrm{CDCl}_{3}\right)$ 158.6, 145.5, 141.3, 129.6, 128.3, 128.0, 126.7, 124.8, 123.8, 123.4, 122.2, 117.8, 115.6, 110.2, 68.4, 31.6, 29.2, 25.7, 22.6, 14.0. MS (FAB, $70 \mathrm{eV}): \mathrm{m} / \mathrm{z}$ (relative intensity) $507\left(\mathrm{M}^{+}, 100\right)$; HRMS calcd for $\mathrm{C}_{32} \mathrm{H}_{29} \mathrm{NOS}_{2}: 507.1691$, found 507.1718.

3,6-bis(5-bromothiophen-2-yl)-9-(4-(hexyloxy)phenyl)-9H-carbazole (4). Compound 4 was synthesised using a method similar to that of 2, producing a yellow solid in a $91.0 \%$ yield. $\delta_{\mathrm{H}}(400$ $\left.\mathrm{MHz} \mathrm{CDCl}_{3}\right) 8.25$ (d, 2H, $\left.J=1.44 \mathrm{~Hz}\right), 7.56(\mathrm{dd}, 2 \mathrm{H}, J=4.30,1.76 \mathrm{~Hz}), 7.38-7.42(\mathrm{~m}, 2 \mathrm{H}), 7.29$ (d, $2 \mathrm{H}, J=8.56 \mathrm{~Hz}), 7.05-7.12(\mathrm{~m}, 6 \mathrm{H}), 4.06(\mathrm{t}, 2 \mathrm{H}, J=6.52 \mathrm{~Hz}), 1.83-1.90(\mathrm{~m}, 2 \mathrm{H}), 1.37-1.56(\mathrm{~m}, 2 \mathrm{H})$, $1.37-1.43(\mathrm{~m}, 4 \mathrm{H}), 0.93-0.96(\mathrm{~m}, 3 \mathrm{H}) . \delta_{\mathrm{C}}\left(100 \mathrm{MHz}, \mathrm{CDCl}_{3}\right) 158.7,146.8,141.5,130.8,129.3,128.2$, 126.0, 124.5, 123.3, 122.3, 117.5, 115.6, 110.4, 110.1, 68.4, 31.5, 29.2, 25.7, 22.6, 14.0. MS (FAB, 70 $\mathrm{eV}): \mathrm{m} / \mathrm{z}$ (relative intensity) $662\left(\mathrm{M}^{+}, 100\right)$; HRMS calcd for $\mathrm{C}_{32} \mathrm{H}_{27}{ }^{79} \mathrm{Br}_{2} \mathrm{NOS}_{2}: 662.9901$, found 662.9901 .

5',5'"-(9-(4-(hexyloxy)phenyl)-9H-carbazole-3,6-diyl)bis(([2,2'-bithiophene]-5-carbaldehyde))

(5). Compound 5 was synthesised using a method similar to that of $\mathbf{3}$, producing a red solid in a $62 \%$ 
yield. $\delta_{\mathrm{H}}\left(400 \mathrm{MHz}, \mathrm{CDCl}_{3}\right) 9.87(\mathrm{~s}, 2 \mathrm{H}), 8.39(\mathrm{~d}, 2 \mathrm{H}, J=1.56 \mathrm{~Hz}), 7.67-7.70(\mathrm{~m}, 4 \mathrm{H}), 7.44-7.45(\mathrm{~m}$, 2H), $7.38(\mathrm{~d}, 2 \mathrm{H}, J=3.84 \mathrm{~Hz}), 7.34(\mathrm{~s}, 2 \mathrm{H}), 7.33$ (d, 2H, $J=4.6 \mathrm{~Hz}), 7.29$ (d, 2H, J=3.92Hz), 7.11$7.15(\mathrm{~m}, 2 \mathrm{H}), 4.08(\mathrm{t}, 2 \mathrm{H}, J=6.56 \mathrm{~Hz}), 1.84-1.91(\mathrm{~m}, 2 \mathrm{H}), 1.50-1.57(\mathrm{~m}, 2 \mathrm{H}), 1.38-1.42(\mathrm{~m}, 4 \mathrm{H})$, $0.93-0.96(\mathrm{~m}, 3 \mathrm{H}) . \delta \mathrm{C}\left(100 \mathrm{MHz}, \mathrm{CDCl}_{3}\right) 182.4,158.9,147.5,147.3,141.8,141.2,137.5,134.1,129.1$, $128.3,127.3,125.9,124.7,123.7,123.4,123.3,117.8,115.7,110.6,68.4,31.6,29.2,25.7,22.6,14.0$. MS (FAB, $70 \mathrm{eV}): \mathrm{m} / \mathrm{z}$ (relative intensity) $727\left(\mathrm{M}^{+}, 100\right)$; HRMS calcd for $\mathrm{C}_{42} \mathrm{H}_{33} \mathrm{NO}_{3} \mathrm{~S}_{4}: 727.1343$, found 727.1350 .

4,4'-((9-(4-(hexyloxy)phenyl)-9H-carbazole-3,6-diyl)bis(thiophene-5,2-diyl))dibenzaldehyde (6). The 4-(4,4,5,5-tetramethyl-1,3,2-dioxaborolan-2-yl)benzaldehyde (2.10 g, $\left.9.06 \mathrm{mmol}), \mathrm{Pd}_{(\mathrm{PPh}}\right)_{4}(7$ $\mathrm{mg}, 0.06 \mathrm{mmol}), 2 \mathrm{M} \mathrm{K}_{2} \mathrm{CO}_{3}(6.8 \mathrm{~mL})$ and compounds $4(2.00 \mathrm{~g}, 3.02 \mathrm{mmol})$ were mixed under a nitrogen atmosphere and stirred in $\mathrm{THF} /$ Toluene at $90{ }^{\circ} \mathrm{C}$ for $18 \mathrm{hr}$. The product was extracted using methylene chloride, and the organic layer was dried over anhydrous $\mathrm{MgSO}_{4}$ and evaporated under vacuum. The product was purified using a silica gel column chromatograph, with ethylene chloride as the eluent. A yellow solid $(1.58 \mathrm{~g}, 25.4 \mathrm{mmol})$ was obtained in a $73 \%$ yield. $\delta_{\mathrm{H}}\left(400 \mathrm{MHz}, \mathrm{CDCl}_{3}\right)$ $9.99(\mathrm{~s}, 2 \mathrm{H}), 8.40(\mathrm{~d}, 2 \mathrm{H}, J=1.52 \mathrm{~Hz}), 7.88-7.90(\mathrm{~m}, 4 \mathrm{H}), 7.79(\mathrm{~d}, 4 \mathrm{H}, J=8.28), 7.70(\mathrm{dd}, 2 \mathrm{H}, J=$ $8.48,1.80 \mathrm{~Hz}), 7.48(\mathrm{~d}, 2 \mathrm{H}, J=3.80), 7.41-7.45(\mathrm{~m}, 2 \mathrm{H}), 7.39(\mathrm{~d}, 2 \mathrm{H}, J=3.88 \mathrm{~Hz}), 7.33(\mathrm{~d}, 2 \mathrm{H}, J=$ $8.52 \mathrm{~Hz}), 7.10-7.14(\mathrm{~m}, 2 \mathrm{H}), 4.07(\mathrm{t}, 2 \mathrm{H}, J=6.48 \mathrm{~Hz}), 1.83-1.91(\mathrm{~m}, 2 \mathrm{H}), 1.50-1.55(\mathrm{~m}, 2 \mathrm{H}), 1.37-$ $1.44(\mathrm{~m}, 4 \mathrm{H}), 0.93-0.97(\mathrm{~m}, 3 \mathrm{H}) . \delta_{\mathrm{C}}\left(100 \mathrm{MHz}, \mathrm{CDCl}_{3}\right) 162.77,158.80,153.95,147.26,141.67,140.56$, $139.18,132.01,129.92,129.25,128.26,126.31,126.22,125.51,124.62,123.62,123.45,117.71$, $115.90,115.72,110.49,101.45,68.45,62.63,31.60,29.24,25.76,22.63,14.18,14.05$. MS (FAB, 70 $\mathrm{eV}): \mathrm{m} / \mathrm{z}$ (relative intensity) $715\left(\mathrm{M}^{+}, 100\right)$; HRMS calcd for $\mathrm{C}_{46} \mathrm{H}_{37} \mathrm{NO}_{3} \mathrm{~S}_{2}: 715.2215$, found 715.2208 .

\section{Diethyl 3,3'-((9-(4-(hexyloxy)phenyl)-9H-carbazole-3,6-diyl)bis(([2,2'-bithiophene]-5',5-diyl)))-} (2E,2'E)-bis(2-cyanoacrylate) (SY-1). A mixture of 5 (1.00 g, $1.38 \mathrm{mmol})$, Ethyl cyanoacetate (47 $\mathrm{mg}, 4.1 \mathrm{mmol}$ ), Acetic acid (3 drops), and Ammonium acetate $(27 \mathrm{mg}, 0.35 \mathrm{mmol}$ ) in toluene was placed in a three-necked flask and stirred at $120^{\circ} \mathrm{C}$ for $18 \mathrm{~h}$. After cooling, the reaction was quenched by adding saturated water, and extracted with ethylene chloride. The organic layer was dried over anhydrous $\mathrm{MgSO}_{4}$ and evaporated under vacuum. The product was purified by silica gel column chromatograph eluted with ethylene chloride. The red solid was isolated in $84.1 \%$ yield (1.06 g, 1.16 mmol). $\delta \mathrm{H}\left(400 \mathrm{MHz}, \mathrm{CDCl}_{3}\right) 8.33(\mathrm{~s}, 2 \mathrm{H}), 8.24(\mathrm{~s}, 2 \mathrm{H}), 7.64(\mathrm{~d}, 4 \mathrm{H}, J=4.6 \mathrm{~Hz}), 7.39-7.42(\mathrm{~m}, 4 \mathrm{H})$, $7.29-7.33(\mathrm{~m}, 4 \mathrm{H}), 7.26(\mathrm{~d}, 2 \mathrm{H}, J=3.92 \mathrm{~Hz}), 7.12(\mathrm{~d}, 2 \mathrm{H}, J=8.72 \mathrm{~Hz}), 4.36(\mathrm{q}, 4 \mathrm{H}, J=7.08 \mathrm{~Hz}), 4.07$ $(\mathrm{t}, 2 \mathrm{H}, J=6.52 \mathrm{~Hz}), 1.83-1.90(\mathrm{~m}, 2 \mathrm{H}), 1.51-1.57(\mathrm{~m}, 2 \mathrm{H}), 1.37-1.43(\mathrm{~m}, 10 \mathrm{H}), 0.93-0.96(\mathrm{~m}, 3 \mathrm{H}) . \delta \mathrm{C}$ $\left(100 \mathrm{MHz} \mathrm{CDCl}_{3}\right)$ 163.0, 158.8, 147.9, 147.7, 146.1, 141.7, 139.4, 133.8, 133.7, 129.1, 128.2, 127.6, $125.8,124.5,123.7,123.4,117.5,116.2$, 115.7, 110.5, 97.1, 68.4, 62.3, 31.6, 29.6, 29.2, 25.7, 22.6, 14.2, 14.0. MS (FAB, $70 \mathrm{eV}): \mathrm{m} / \mathrm{z}$ (relative intensity) $917\left(\mathrm{M}^{+}, 100\right)$; HRMS calcd for $\mathrm{C}_{52} \mathrm{H}_{43} \mathrm{~N}_{3} \mathrm{O}_{5} \mathrm{~S}_{4}$ : 917.2086, found 917.2079 . 
(2E,2'E)-3,3'-((9-(4-(hexyloxy)phenyl)-9H-carbazole-3,6-diyl)bis([2,2'-bithiophene]-5',5-diyl))bis(2-cyano-N-hexylacrylamide) (SY-2). A mixture of 5 (1.00 g, $1.38 \mathrm{mmol})$, 2-cyano-Nhexylacetamide (700 mg, $4.14 \mathrm{mmol}$ ), Acetic acid (3 drops), and Ammonium acetate (27 $\mathrm{mg}, 0.35$ $\mathrm{mmol}$ ) in toluene was placed in a three-necked flask and stirred at $120^{\circ} \mathrm{C}$ for $18 \mathrm{~h}$. After cooling, the reaction was quenched by adding saturated water, and extracted with ethylene chloride. The organic layer was dried over anhydrous $\mathrm{MgSO}_{4}$ and evaporated under vacuum. The product was purified by silica gel column chromatograph eluted with ethylene chloride. The red solid was isolated in $76.1 \%$ yield (1.08 g, $1.05 \mathrm{mmol}) . \delta_{\mathrm{H}}\left(400 \mathrm{MHz}, \mathrm{CDCl}_{3}\right) 8.33(\mathrm{~s}, 2 \mathrm{H}), 8.24(\mathrm{~s}, 2 \mathrm{H}), 7.64(\mathrm{~d}, 4 \mathrm{H}, J=4.6 \mathrm{~Hz})$, 7.39-7.42 (m, 4H), 7.29-7.33 (m, 4H), $7.26(\mathrm{~d}, 2 \mathrm{H}, J=3.92 \mathrm{~Hz}), 7.12(\mathrm{~d}, 2 \mathrm{H}, J=8.72 \mathrm{~Hz}), 4.36(\mathrm{q}$, $4 \mathrm{H}, J=7.08 \mathrm{~Hz}), 4.07(\mathrm{t}, 2 \mathrm{H}, J=6.52 \mathrm{~Hz}), 1.83-1.90(\mathrm{~m}, 2 \mathrm{H}), 1.51-1.57(\mathrm{~m}, 2 \mathrm{H}), 1.37-1.43(\mathrm{~m}, 10 \mathrm{H})$, 0.93-0.96 (m, 3H). $\delta_{\mathrm{C}}\left(100 \mathrm{MHz}, \mathrm{CDCl}_{3}\right) 163.0,158.8,147.9,147.7,146.1,141.7,139.4,133.8,133.7$, 129.1, 128.2, 127.6, 125.8, 124.5, 123.7, 123.4, 117.5, 116.2, 115.7, 110.5, 97.1, 68.4, 62.3, 31.6, 29.6, 29.2, 25.7, 22.6, 14.2, 14.0. MS (FAB, $70 \mathrm{eV}): \mathrm{m} / \mathrm{z}$ (relative intensity) $1027\left(\mathrm{M}^{+}, 100\right)$; HRMS calcd for $\mathrm{C}_{60} \mathrm{H}_{61} \mathrm{~N}_{5} \mathrm{O}_{3} \mathrm{~S}_{4}: 1027.3657$ found 1027.3658 .

Diethyl 3,3'-((9-(4-(hexyloxy)phenyl)-9H-carbazole-3,6-diyl)bis(thiophene-5,2-diyl))bis(4,1phenylene))(2E,2'E)-bis(2-cyanoacrylate) (SY-3). Compound SY-3 was synthesised using a method similar to that of SY-1, producing a red solid in a $92.2 \%$ yield. $\delta_{\mathrm{H}}\left(400 \mathrm{MHz}, \mathrm{CDCl}_{3}\right) 8.40(\mathrm{~d}, 2 \mathrm{H}, J=$ $1.4 \mathrm{~Hz}), 8.20$ (s, 2H), 8.02 (d, 2H, $J=8.48 \mathrm{~Hz}), 7.75$ (d, 4H, $J=8.44 \mathrm{~Hz}), 7.69$ (dd, 2H, $J=8.64,1.56$ $\mathrm{Hz}), 7.49$ (d, 2H, $J=3.80 \mathrm{~Hz}), 7.43(\mathrm{~d}, 2 \mathrm{H}, J=8.76 \mathrm{~Hz}), 7.39(\mathrm{~d}, 2 \mathrm{H}, J=3.84 \mathrm{~Hz}), 7.33$ (d, 2H, $J=$ $8.56 \mathrm{~Hz}), 7.12(\mathrm{~d}, 2 \mathrm{H}, J=8.84 \mathrm{~Hz}), 4.39$ (q, 4H, $J=7.20 \mathrm{~Hz}), 4.07$ (t , 2H, $J=6.48 \mathrm{~Hz}), 1.83-1.90$ (m, $2 \mathrm{H}), 1.51-1.58(\mathrm{~m}, 2 \mathrm{H}), 1.38-1.43(\mathrm{~m}, 10 \mathrm{H}), 0.93-0.96(\mathrm{~m}, 3 \mathrm{H}) . \delta_{\mathrm{C}}\left(100 \mathrm{MHz}, \mathrm{CDCl}_{3}\right) 162.7,158.8$, $153.9,147.2,141.6,140.5,139.1,132.0,129.9,129.2,128.2,126.3,126.2,125.5,124.6,123.6,123.4$, $117.7,115.9,115.7,110.4,101.4,68.4,62.6,31.6,29.2,25.7,22.6,14.1,14.0 . \mathrm{MS}(\mathrm{FAB}, 70 \mathrm{eV}): \mathrm{m} / \mathrm{z}$ (relative intensity) $905\left(\mathrm{M}^{+}, 100\right)$; HRMS calcd for $\mathrm{C}_{56} \mathrm{H}_{47} \mathrm{~N}_{3} \mathrm{O}_{5} \mathrm{~S}_{2}: 905.2957$, found 905.2964.

\section{(2E,2'E)-3,3'-(((9-(4-(hexyloxy)phenyl)-9H-carbazole-3,6-diyl)bis(thiophene-5,2-diyl))bis(4,1-} phenylene))bis(2-cyano-N-hexylacrylamide) (SY-4). Compound SY-4 was synthesised using a method similar to that of $\mathbf{S y - 2}$, producing a orange solid in a $89.4 \%$ yield. $\delta_{\mathrm{H}}\left(400 \mathrm{MHz}, \mathrm{CDCl}_{3}\right) 8.40$ $(\mathrm{d}, 2 \mathrm{H}, J=1.48 \mathrm{~Hz}), 8.29$ (s, 2H), $7.96(\mathrm{~d}, 4 \mathrm{H}, J=8.52 \mathrm{~Hz}), 7.74(\mathrm{~d}, 4 \mathrm{H}, J=8.40 \mathrm{~Hz}), 7.69$ (dd, 2H, $J$ $=8.52,1.72 \mathrm{~Hz}), 7.47(\mathrm{~d}, 2 \mathrm{H}, J=3.8 \mathrm{~Hz}), 7.43(\mathrm{~d}, 2 \mathrm{H}, J=8.76 \mathrm{~Hz}), 7.39(\mathrm{~d}, 2 \mathrm{H}, J=3.8 \mathrm{~Hz}), 7.32(\mathrm{~d}$, $2 \mathrm{H}, J=8.56 \mathrm{~Hz}), 7.12(\mathrm{~d}, 2 \mathrm{H}, J=8.8 \mathrm{~Hz}), 6.36(\mathrm{t}, 2 \mathrm{H}, J=5.68 \mathrm{~Hz}), 4.07(\mathrm{t}, 2 \mathrm{H}, J=6.44 \mathrm{~Hz}), 3.43$ $(\mathrm{q}, 4 \mathrm{H}, J=6.20 \mathrm{~Hz}), 1.83-1.90(\mathrm{~m}, 2 \mathrm{H}), 1.50-1.65(\mathrm{~m}, 6 \mathrm{H}), 1.31-1.42(\mathrm{~m}, 16 \mathrm{H}), 0.89-0.96(\mathrm{~m}, 9 \mathrm{H}) . \delta \mathrm{C}$ $\left(100 \mathrm{MHz}, \mathrm{CDCl}_{3}\right)$ 160.3, 158.7, 151.7, 146.9, 141.6, 140.7, 138.5, 131.4, 130.3, 129.2, 128.2, 126.2, 126.0, 125.5, 124.6, 123.5, 123.4, 117.6, 117.4, 115.7, 110.4, 102.6, 68.4, 40.6, 31.6, 31.4, 29.3, 29.2, 26.5, 25.7, 22.6, 22.5, 14.0, 14.0. MS (FAB, $70 \mathrm{eV}): \mathrm{m} / \mathrm{z}$ (relative intensity) $1015\left(\mathrm{M}^{+}, 100\right)$; HRMS calcd for $\mathrm{C}_{64} \mathrm{H}_{65} \mathrm{~N}_{5} \mathrm{O}_{3} \mathrm{~S}_{2}: 1015.4529$, found 1015.4519 . 
2. ${ }^{1} \mathrm{H}$ and ${ }^{13} \mathrm{C}$ NMR spectra
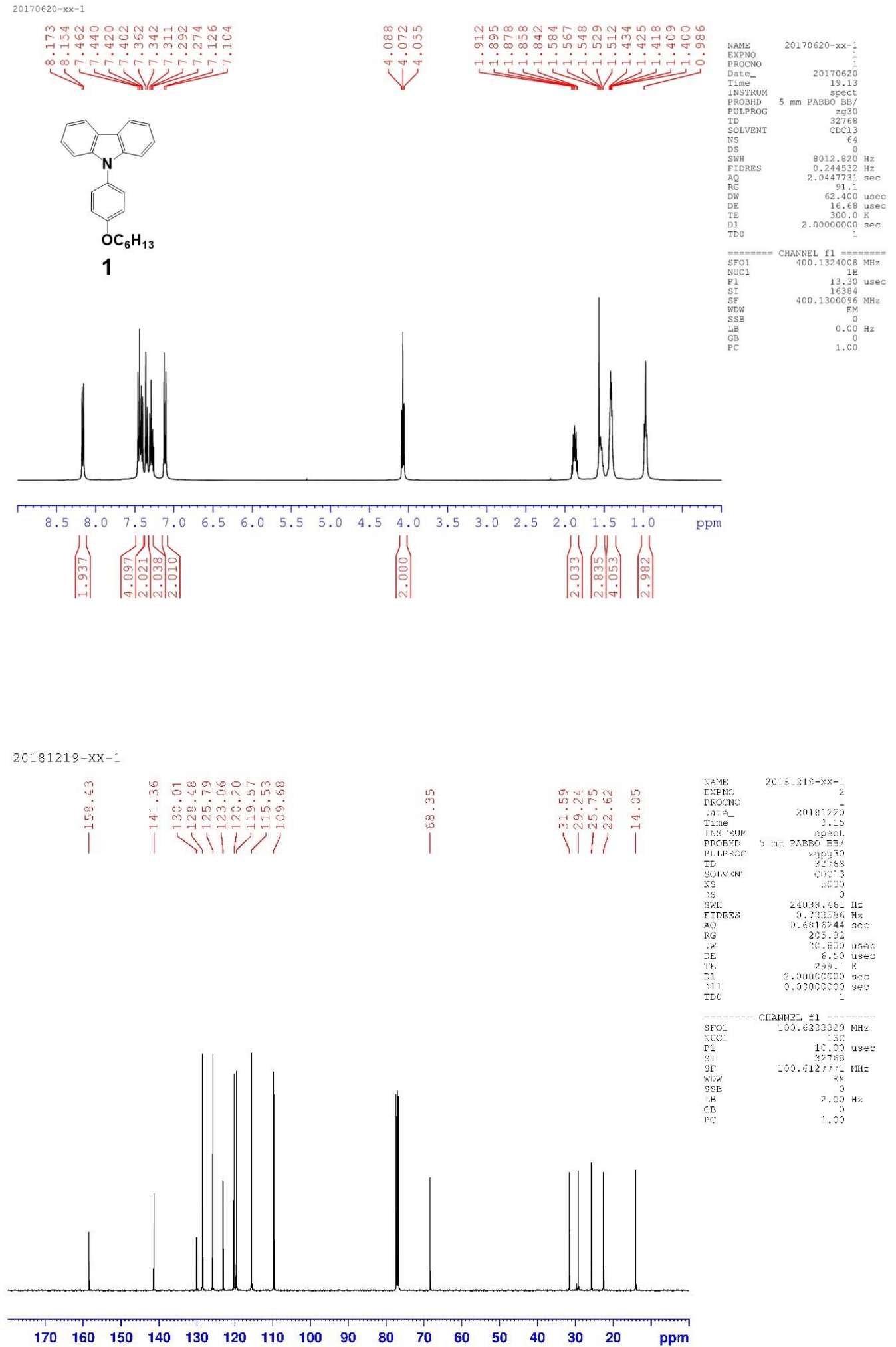

Figure S1. ${ }^{1} \mathrm{H}$ NMR (upper) and ${ }^{13} \mathrm{C}$ NMR (lower) spectra of $\mathbf{1}$ in $\mathrm{CDCl}_{3}$. 

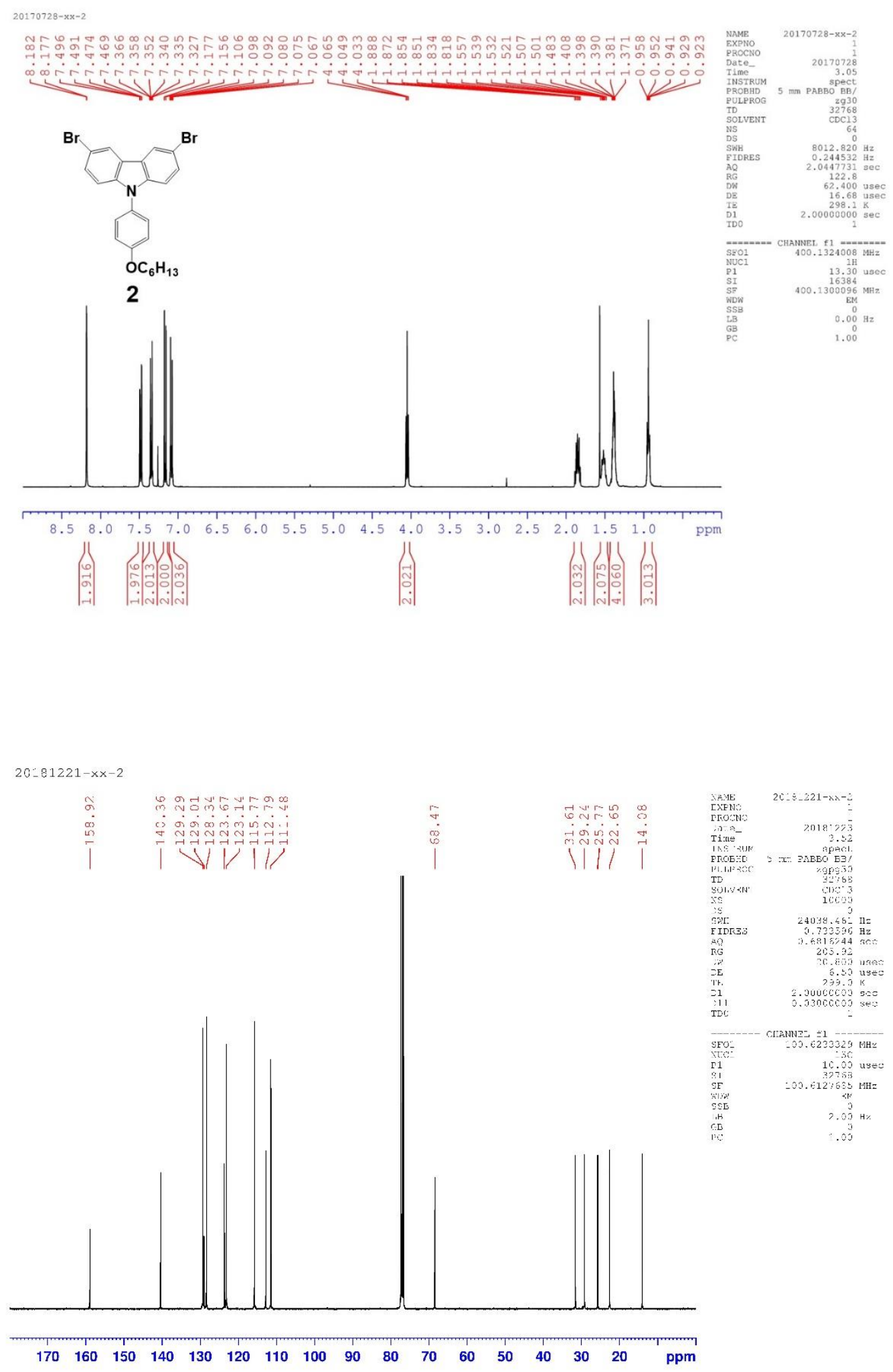

Figure S2. ${ }^{1} \mathrm{H}$ NMR (upper) and ${ }^{13} \mathrm{C}$ NMR (lower) spectra of 2 in $\mathrm{CDCl}_{3}$. 

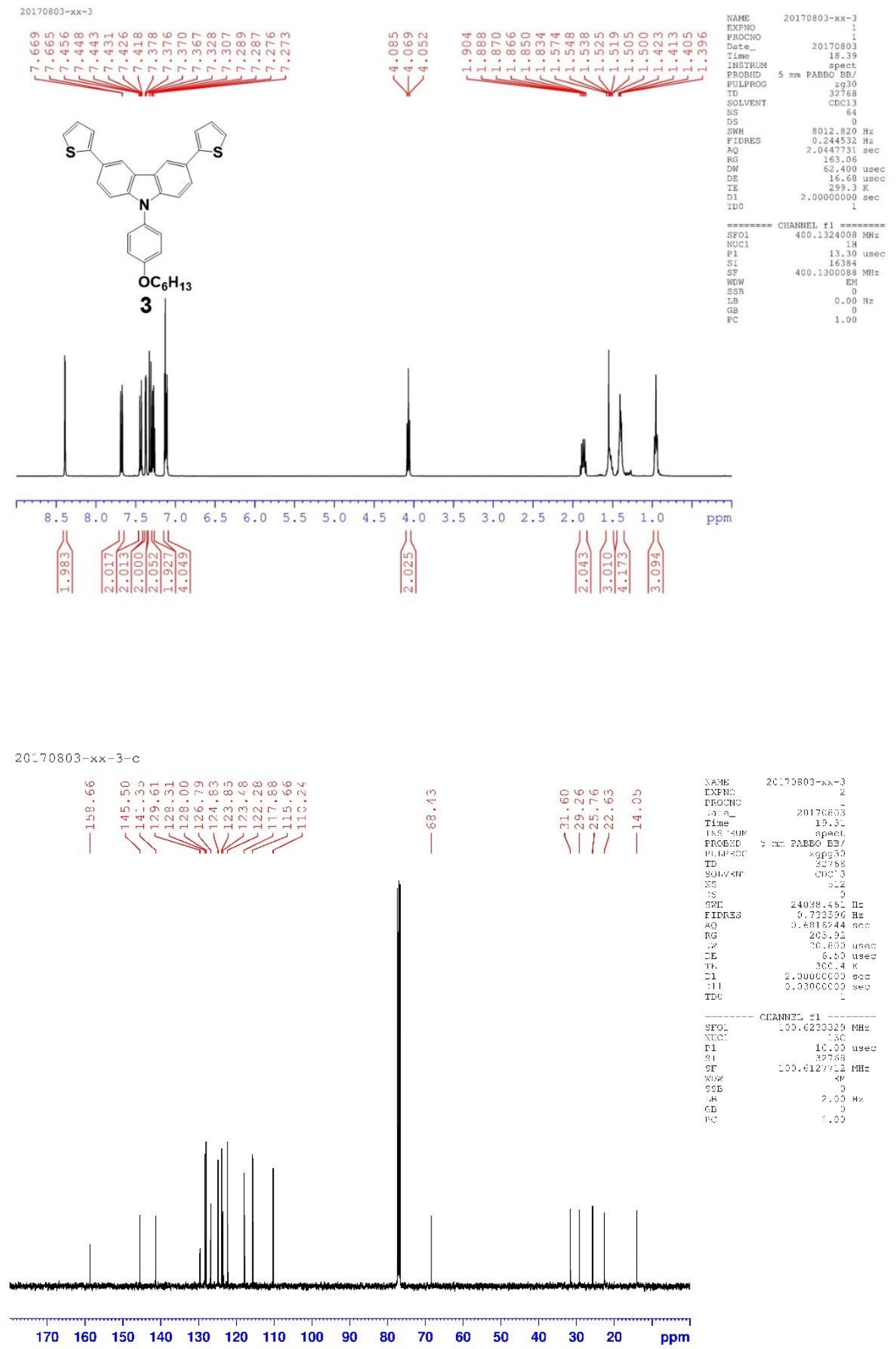

Figure S3. ${ }^{1} \mathrm{H}$ NMR (upper) and ${ }^{13} \mathrm{C} \mathrm{NMR}$ (lower) spectra of $\mathbf{3}$ in $\mathrm{CDCl}_{3}$. 

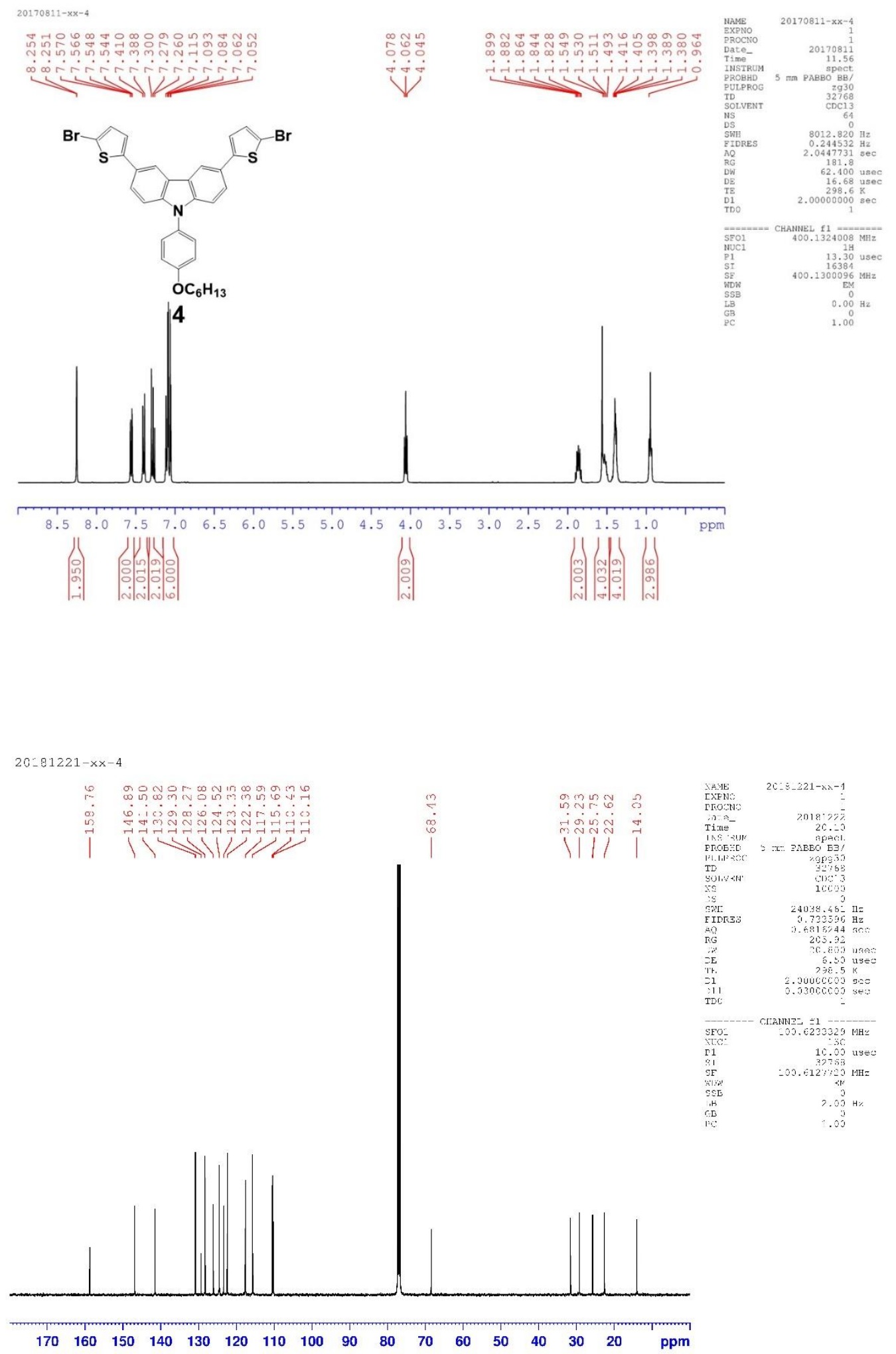

Figure S4. ${ }^{1} \mathrm{H}$ NMR (upper) and ${ }^{13} \mathrm{C} \mathrm{NMR}$ (lower) spectra of 4 in $\mathrm{CDCl}_{3}$. 

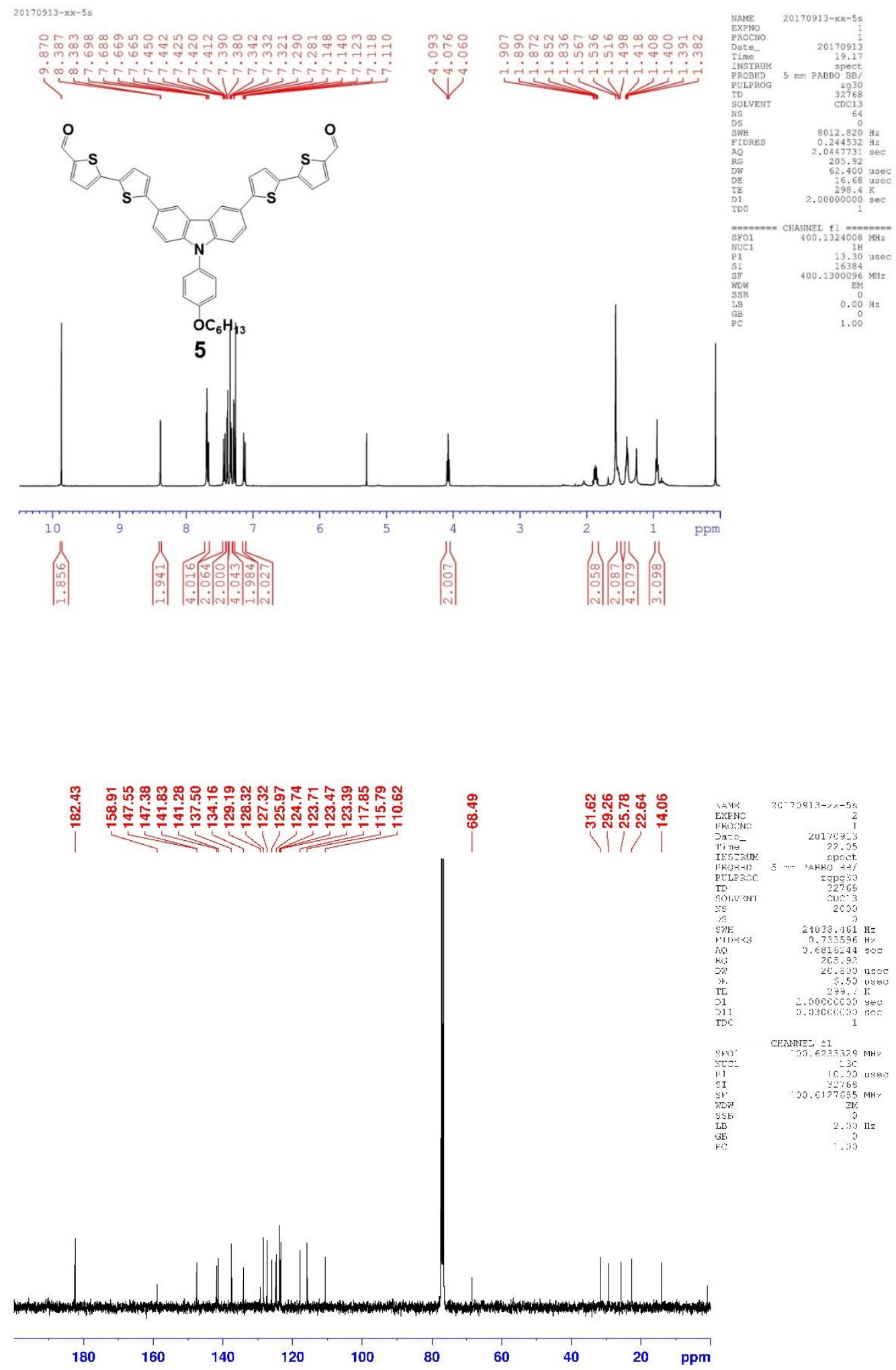

Figure S5. ${ }^{1} \mathrm{H}$ NMR (upper) and ${ }^{13} \mathrm{C}$ NMR (lower) spectra of 5 in $\mathrm{CDCl}_{3}$. 

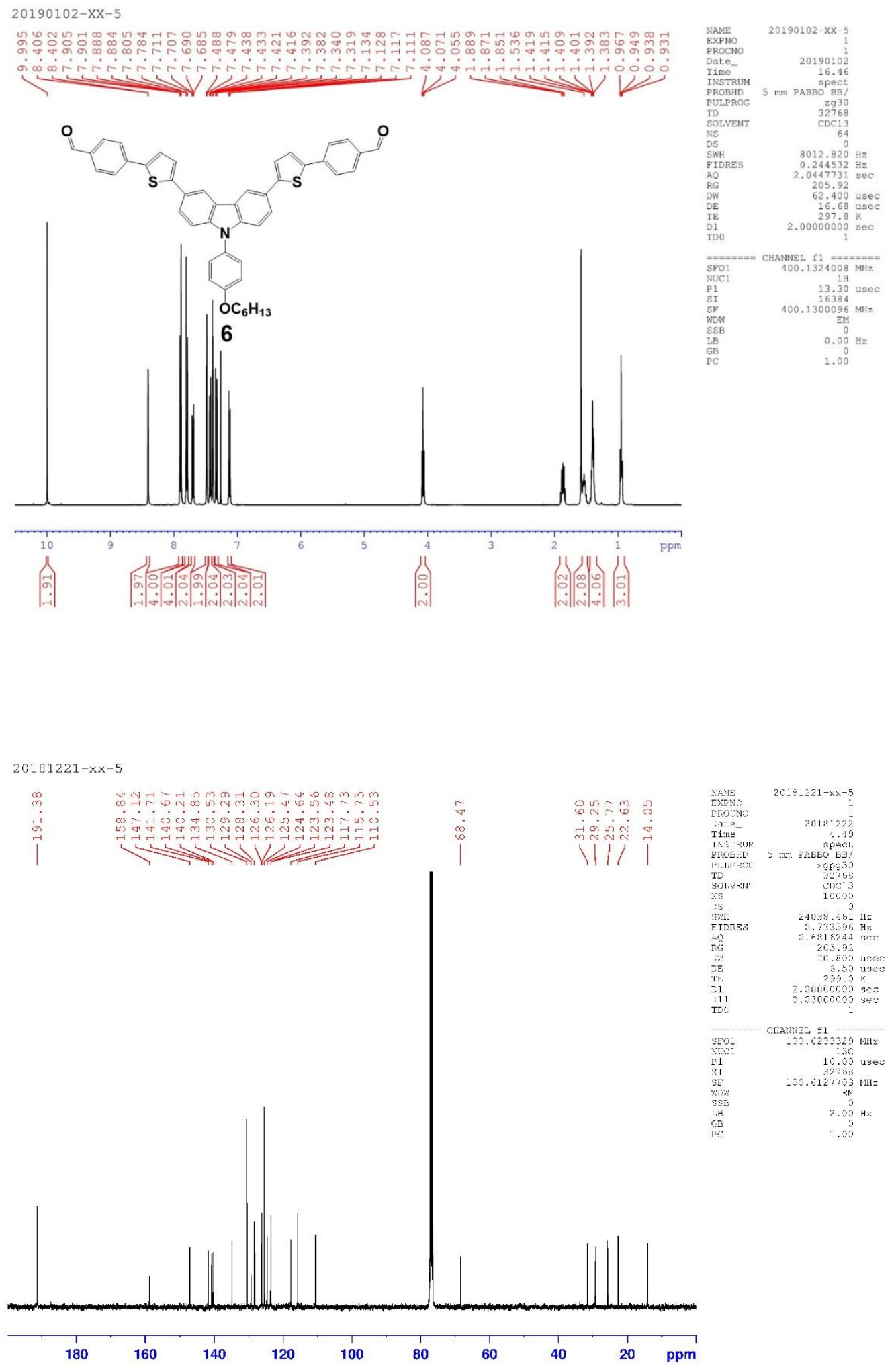

Figure S6. ${ }^{1} \mathrm{H}$ NMR (upper) and ${ }^{13} \mathrm{C} \mathrm{NMR}$ (lower) spectra of 6 in $\mathrm{CDCl}_{3}$. 

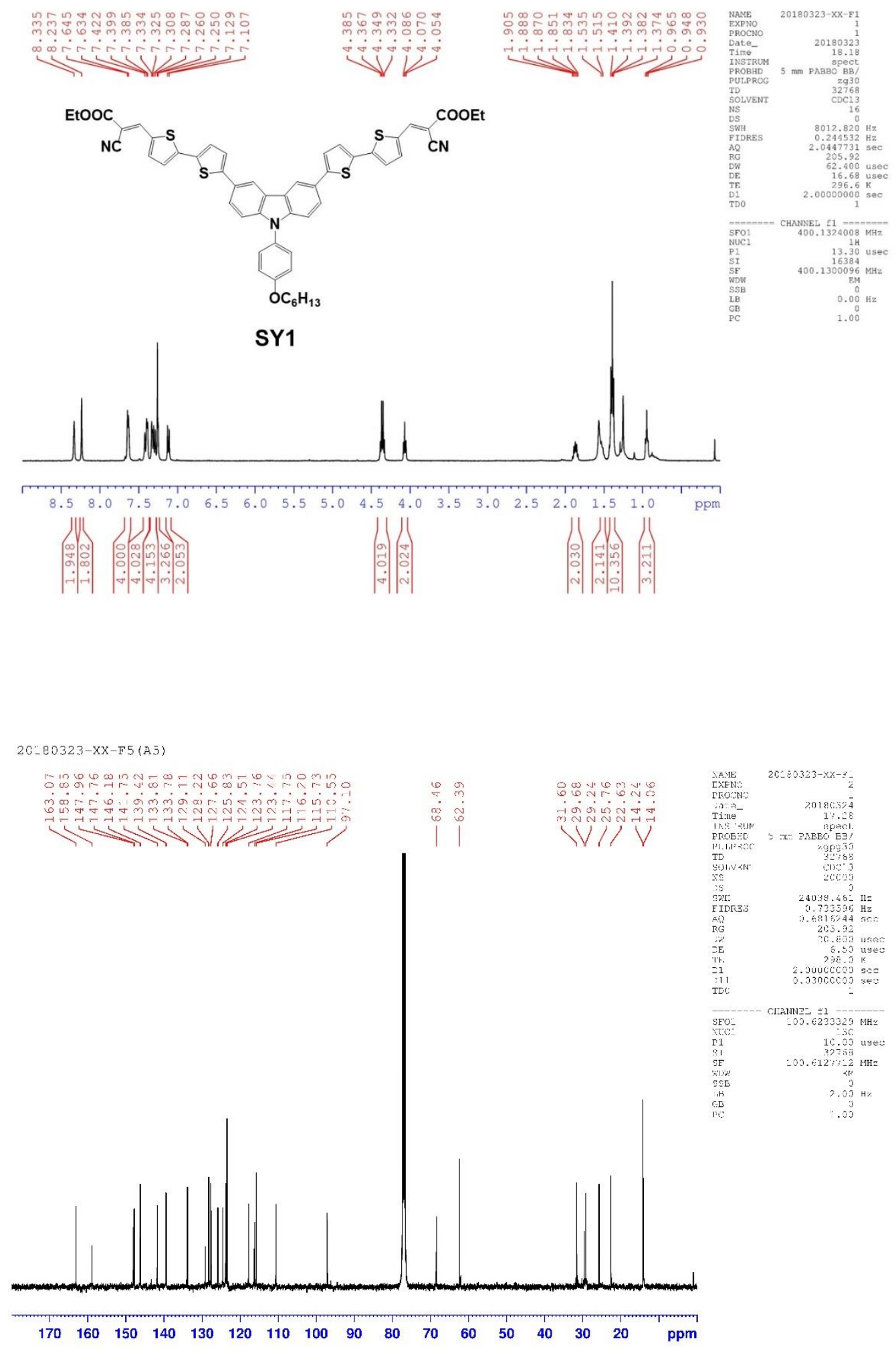

Figure S7. ${ }^{1} \mathrm{H}$ NMR (upper) and ${ }^{13} \mathrm{C}$ NMR (lower) spectra of $\mathbf{S Y} \mathbf{1}$ in $\mathrm{CDCl}_{3}$. 

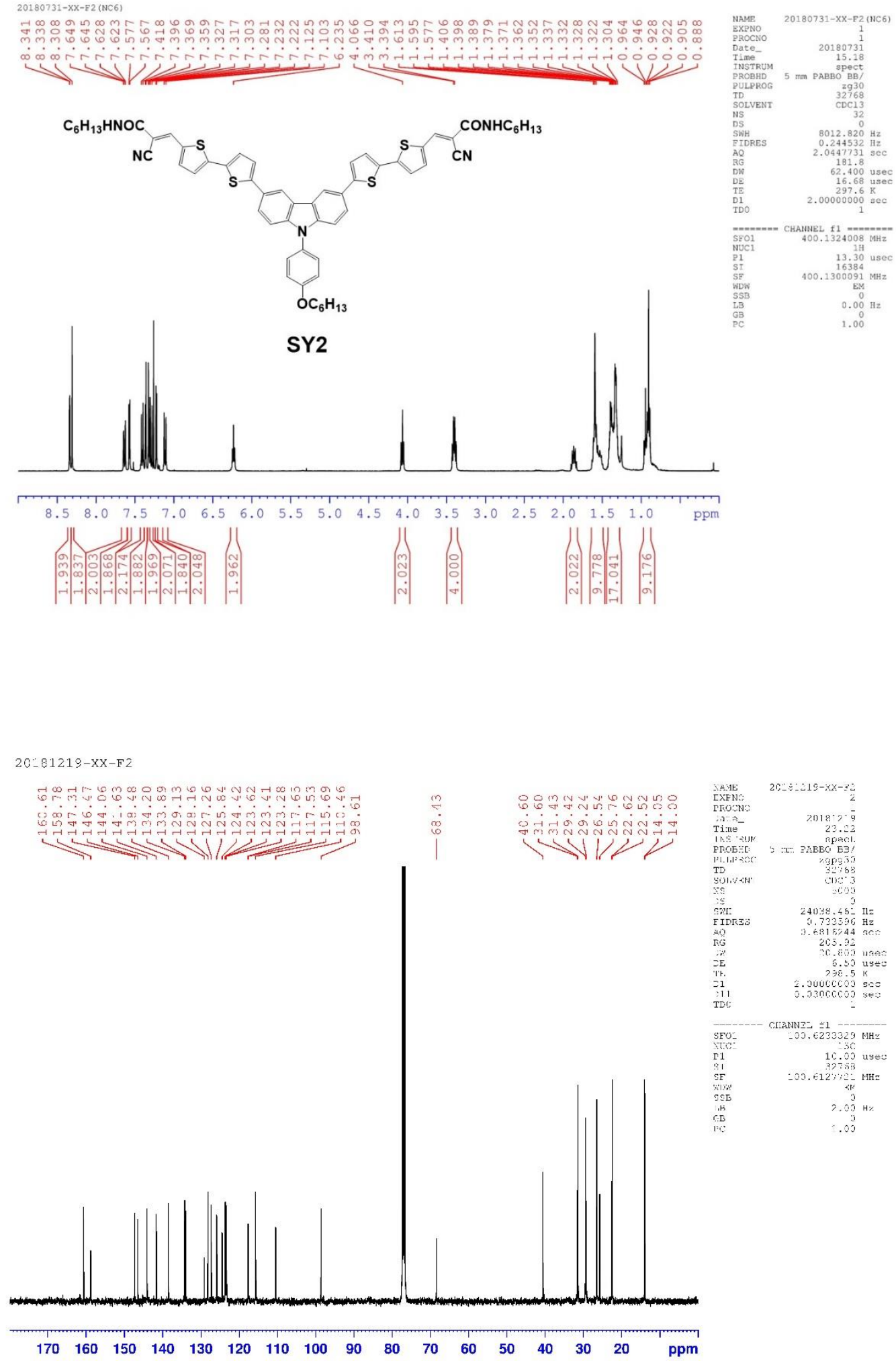

Figure S8. ${ }^{1} \mathrm{H}$ NMR (upper) and ${ }^{13} \mathrm{C}$ NMR (lower) spectra of $\mathbf{S Y 2}$ in $\mathrm{CDCl}_{3}$. 

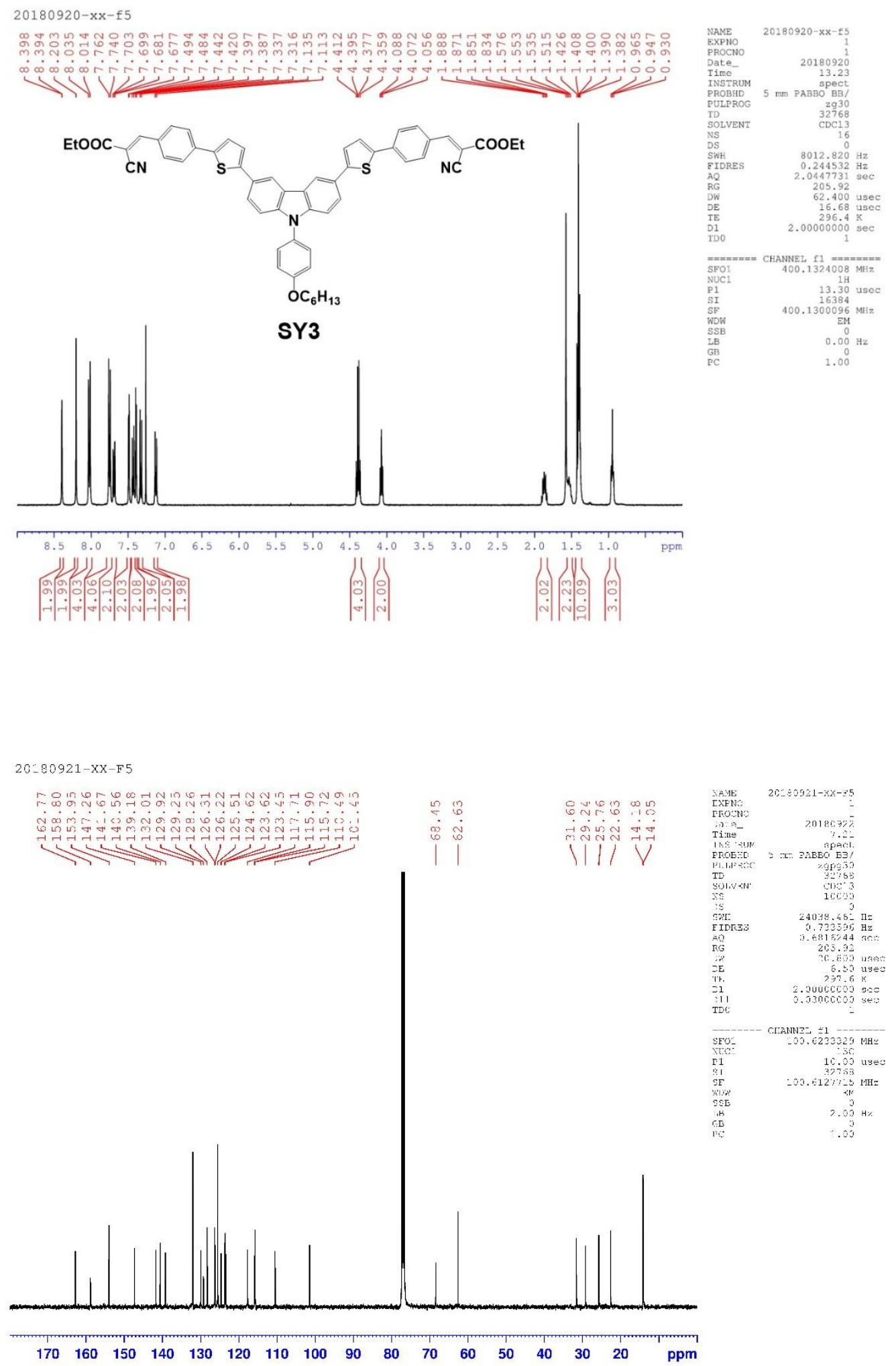

Figure S9. ${ }^{1} \mathrm{H}$ NMR (upper) and ${ }^{13} \mathrm{C}$ NMR (lower) spectra of $\mathbf{S Y 3}$ in $\mathrm{CDCl}_{3}$. 

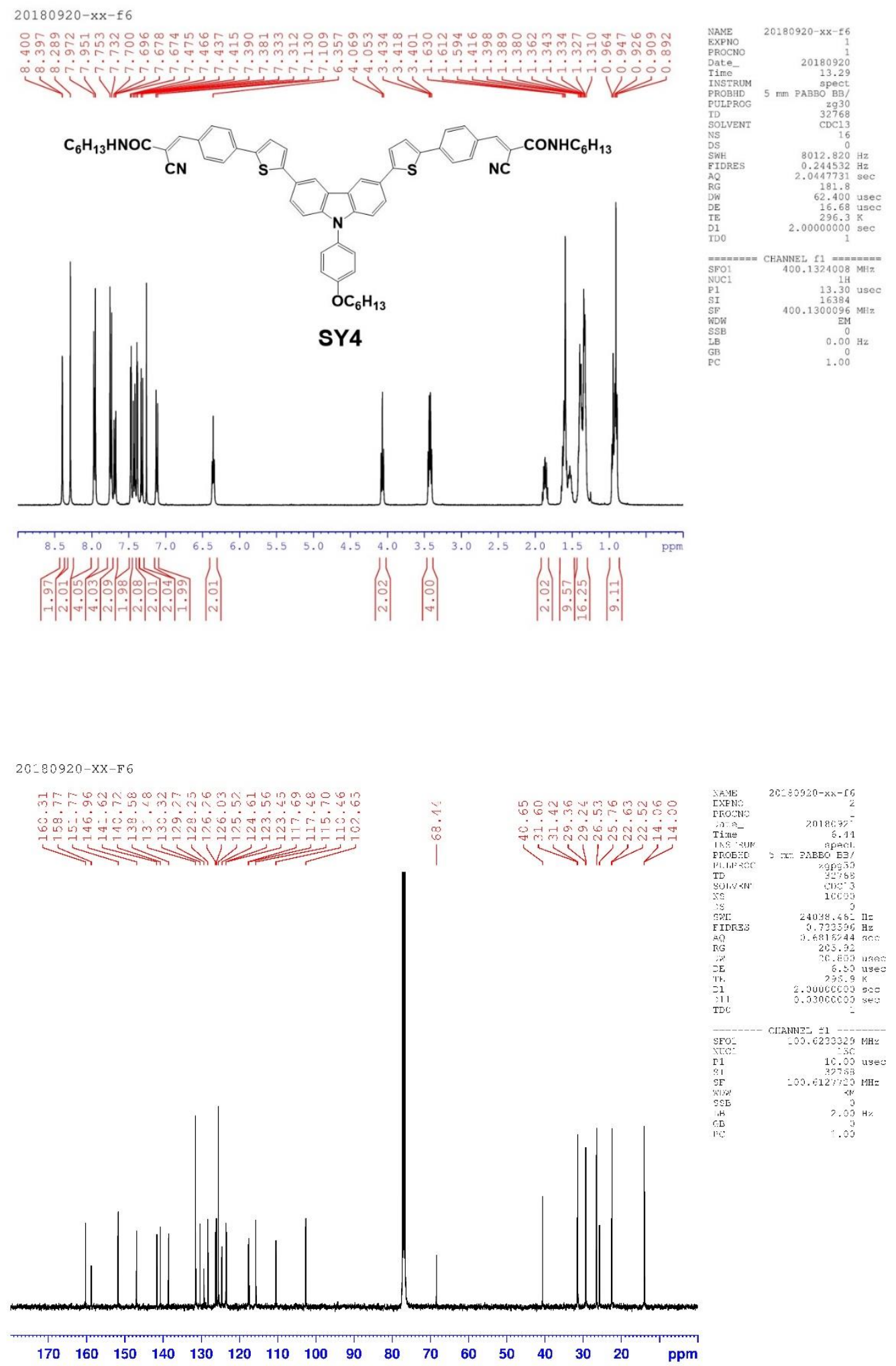

Figure S10. ${ }^{1} \mathrm{H}$ NMR (upper) and ${ }^{13} \mathrm{C}$ NMR (lower) spectra of SY 4 in $\mathrm{CDCl}_{3}$. 
(a)

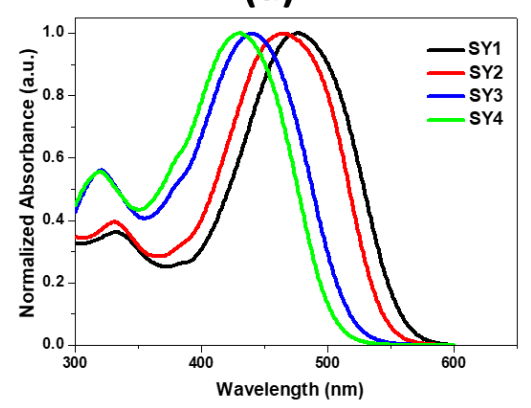

(b)

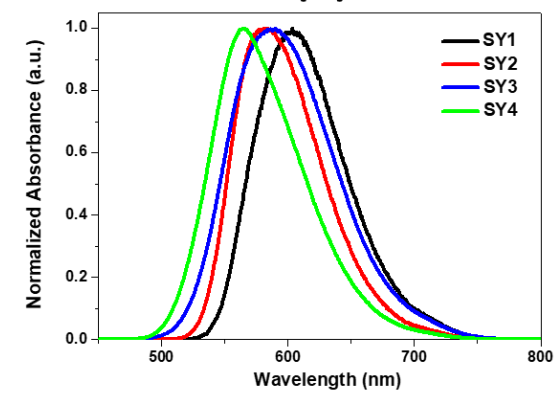

(c)

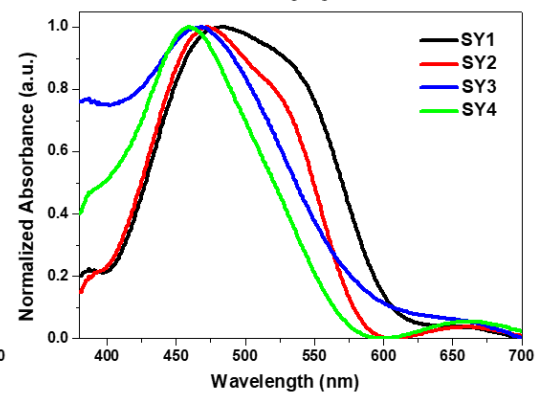

Figure S11. (a) UV-Vis absorption spectra in $\mathrm{CH}_{2} \mathrm{Cl}_{2}$. (b) $\mathrm{PL}$ spectra in $\mathrm{CH}_{2} \mathrm{Cl}_{2}$ (c) UV-Vis absorption spectra in thin film.

4. Oxidative voltammograms of HTMs.

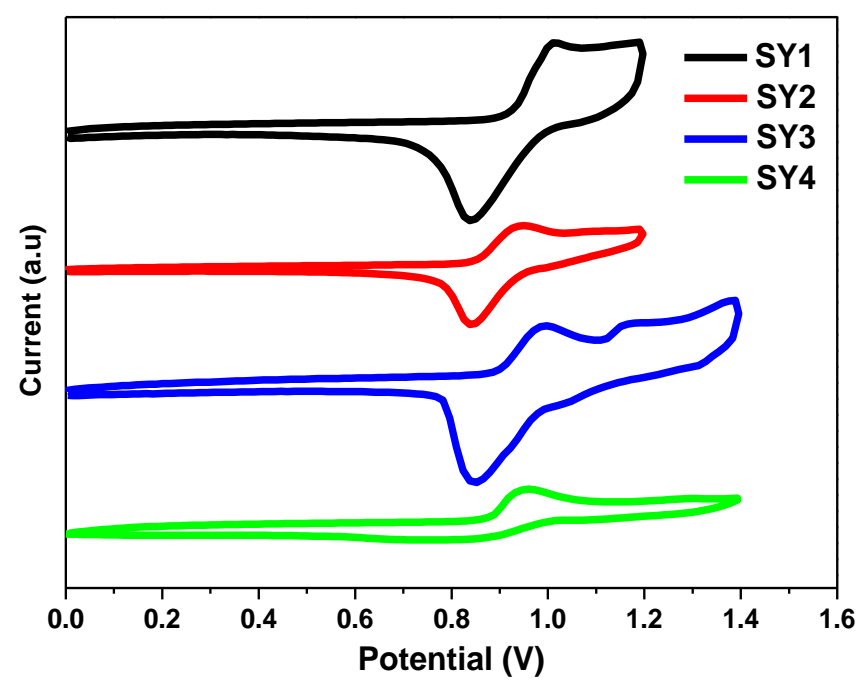

Figure S12. Oxidative voltammograms of SY-HTMs. 


\section{Theoretical calculation}

Table S1. Calculated Low-Lying Transition for SY-series HTMs.

\begin{tabular}{|c|c|c|c|c|c|c|}
\hline \multirow[t]{2}{*}{ HTM } & \multirow{2}{*}{$\begin{array}{c}\text { State } \\
\text { S1 }\end{array}$} & \multicolumn{2}{|c|}{ excitation $^{a}$} & \multirow{2}{*}{$\begin{array}{c}\begin{array}{c}\lambda_{\text {cal }} \\
(\mathrm{eV}, \mathrm{nm})\end{array} \\
2.34(530)\end{array}$} & \multirow{2}{*}{$\begin{array}{c}f^{b} \\
\text { B3LYP/631G* } \\
1.1224\end{array}$} & \multirow[t]{2}{*}{ Dipole moment (D) } \\
\hline & & $70.10 \%$ & $\mathrm{H} \rightarrow \mathrm{L}$ & & & \\
\hline \multirow[t]{3}{*}{ SY1 } & S2 & $68.94 \%$ & $\mathrm{H} \rightarrow \mathrm{L}+1$ & $2.46(503)$ & 0.2597 & 18.7792 \\
\hline & S3 & $69.26 \%$ & $\mathrm{H}-1 \rightarrow \mathrm{L}+1$ & $2.71(457)$ & 0.5962 & \\
\hline & S1 & $69.78 \%$ & $\mathrm{H} \rightarrow \mathrm{L}$ & $2.40(516)$ & 1.1971 & \\
\hline \multirow[t]{3}{*}{ SY2 } & S2 & $69.14 \%$ & $\mathrm{H} \rightarrow \mathrm{L}+1$ & $2.53(489)$ & 0.2791 & 20.0110 \\
\hline & S3 & $65.17 \%$ & $\mathrm{H}-1 \rightarrow \mathrm{L}+1$ & $2.77(447)$ & 0.5961 & \\
\hline & S1 & $69.92 \%$ & $\mathrm{H} \rightarrow \mathrm{L}$ & $2.45(506)$ & 1.2246 & \\
\hline \multirow[t]{3}{*}{ SY3 } & S2 & $70.26 \%$ & $\mathrm{H} \rightarrow \mathrm{L}+1$ & $2.56(484)$ & 0.2285 & 18.4690 \\
\hline & S3 & $67.80 \%$ & $\mathrm{H}-1 \rightarrow \mathrm{L}+1$ & $2.88(430)$ & 0.7081 & \\
\hline & S1 & $69.70 \%$ & $\mathrm{H} \rightarrow \mathrm{L}$ & $2.54(488)$ & 1.3139 & \\
\hline \multirow[t]{2}{*}{ SY4 } & S2 & $70.33 \%$ & $\mathrm{H} \rightarrow \mathrm{L}+1$ & $2.66(465)$ & 0.2417 & 19.2521 \\
\hline & S3 & $64.53 \%$ & $\mathrm{H}-1 \rightarrow \mathrm{L}+1$ & $2.98(416)$ & 0.6848 & \\
\hline
\end{tabular}

(a)

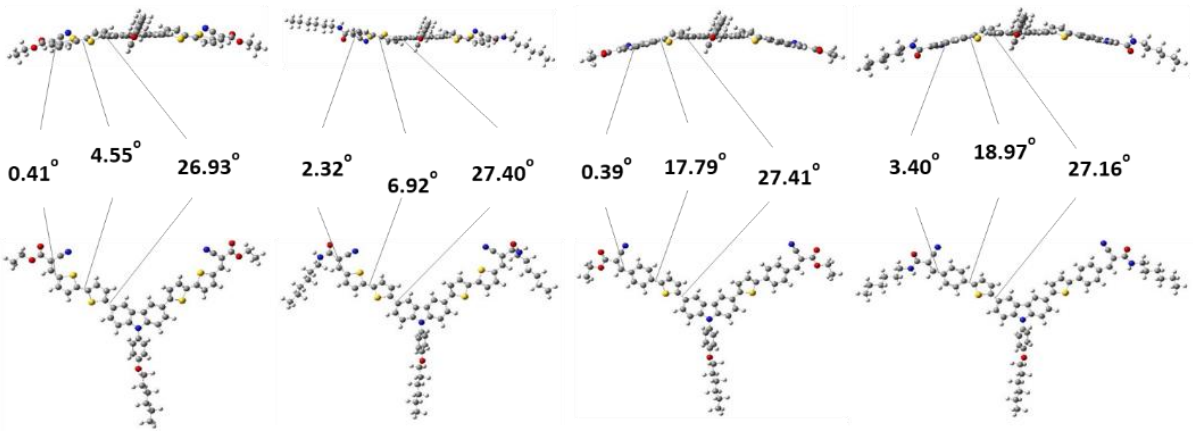

(b)

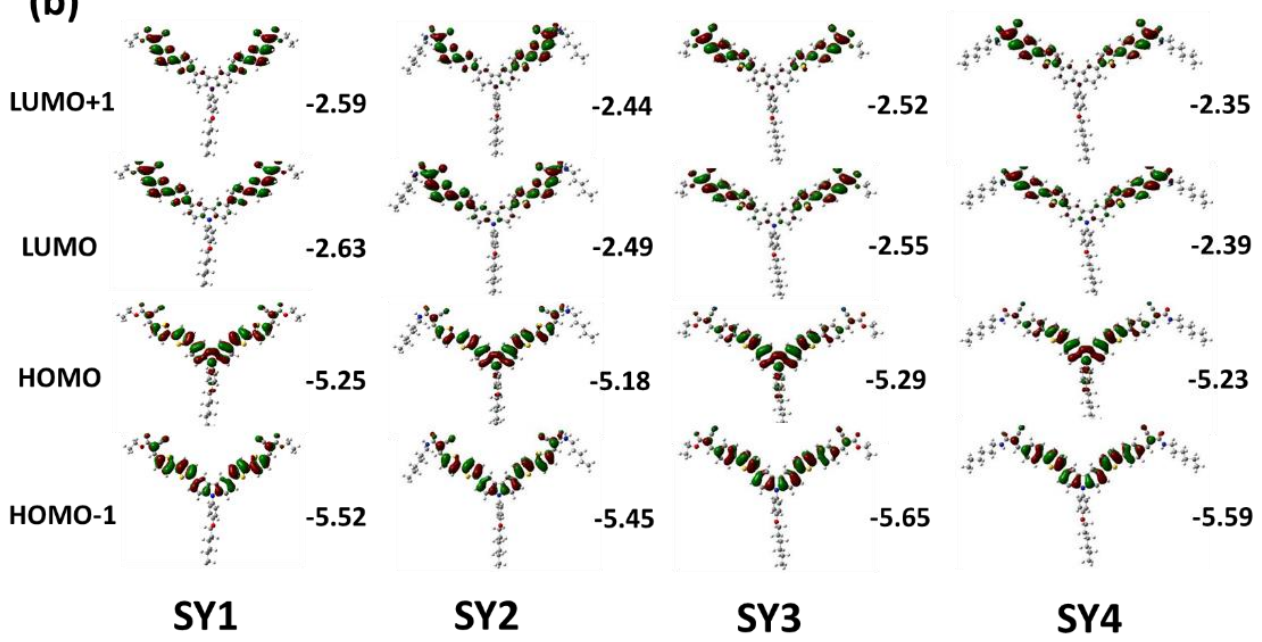

Fig. S13. (a) Computed dihedral angles of SY-series HTMs. (b) Computed HOMO and LUMO energy levels and molecule orbitals of SY-series HTMs. 


\section{Space Charge Limited Current Measurements (SCLC)}

Table S2 The hole mobilities characteristics of SY1 SY4 were measured by space charge limited current (SCLC)measurements .

\begin{tabular}{|c|c|}
\hline HTM & Mobility $\left(\mathrm{cm}^{2} \mathrm{~V}^{-1} \mathrm{~s}^{-1}\right)$ \\
\hline SY1 & $8.19^{*} 10^{-6}$ \\
\hline SY2 & $9.41 * 10^{-5}$ \\
\hline SY3 & $6.79 * 10^{-6}$ \\
\hline SY4 & $1.81 * 10^{-5}$ \\
\hline
\end{tabular}

7. UV, TRPL and PL spectra of PSCs device

(a)

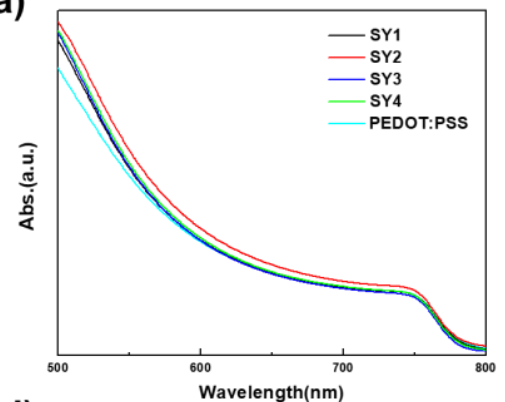

(d)

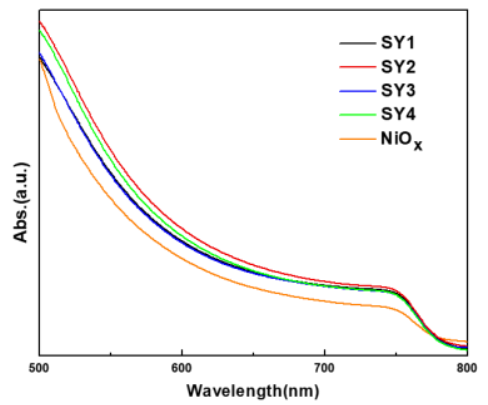

(b)

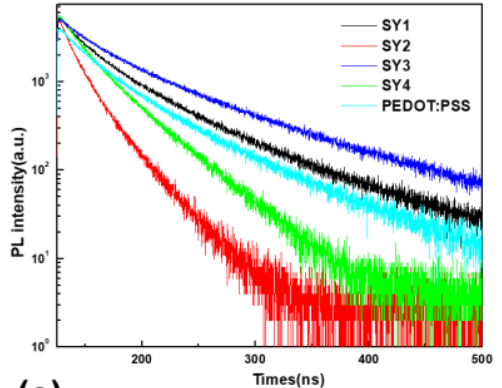

(e)

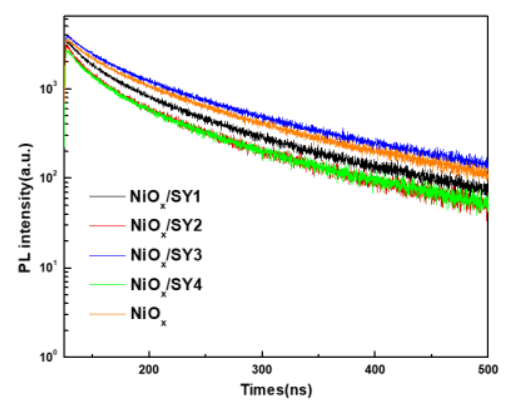

(c)

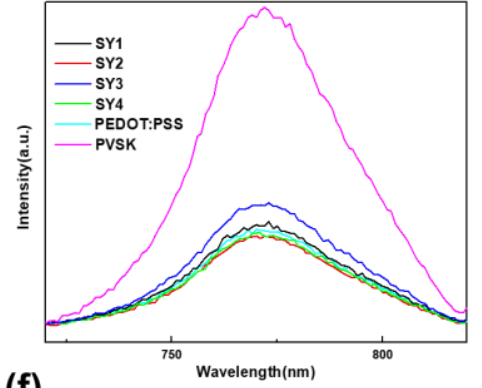

(f)

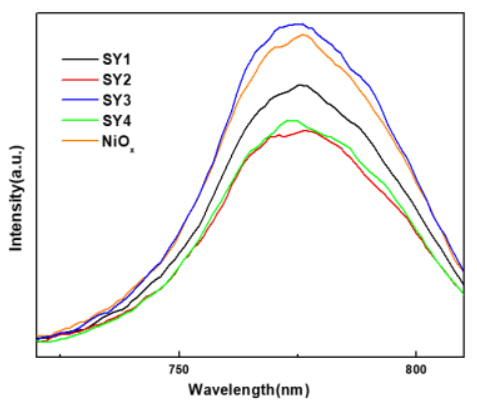

Figure S14. (a, d) UV-Vis, (b, e) TRPL, and (c, f) PL spectra of perovskite films spin-coated on the HTMs 


\section{Contact angle and surface energy}

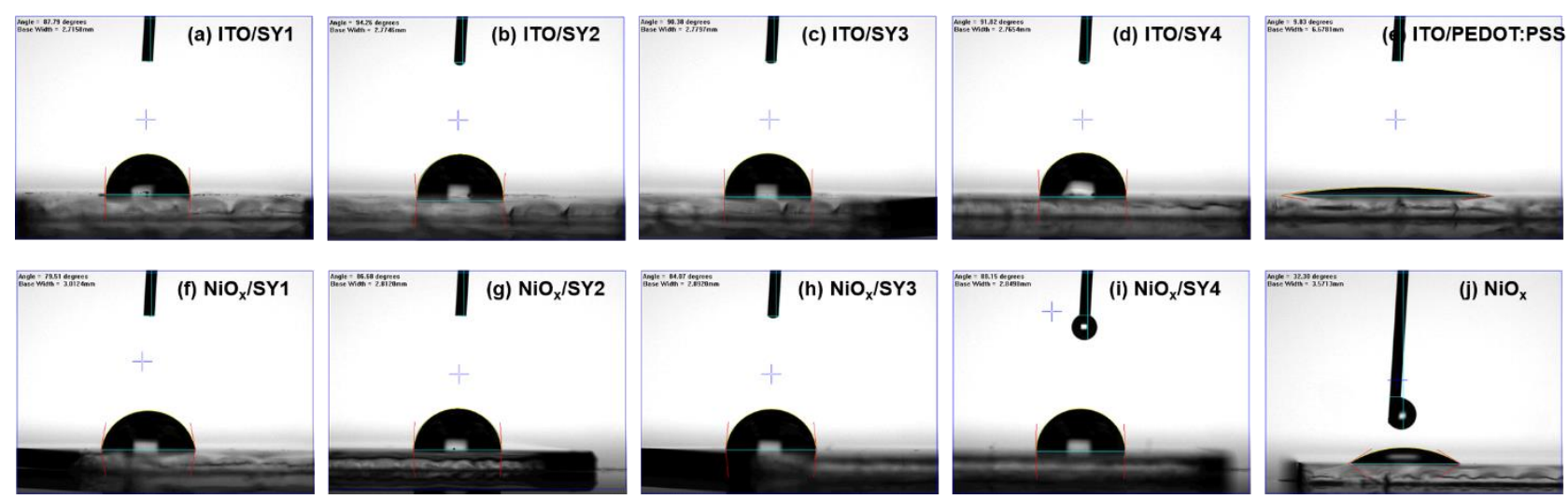

Figure S15(a-j). Water contact angles of SY-HTMs films on ITO and ITO/NiO $\mathrm{N}_{\mathrm{x}}$ substrates.

Table S3. Water contact angles, diiodomethane contact angles, and surface energies of the HTMs films.

\begin{tabular}{cccc}
\hline HTM & $\theta_{\text {water }}\left(^{\circ}\right)$ & $\theta_{\text {Diiodomethane }}\left({ }^{\circ}\right)$ & $\gamma_{\text {total }}\left(\mathrm{mN} \cdot \mathrm{m}^{-1}\right)$ \\
\hline SY1 & 87.79 & 37.11 & 46.53 \\
SY2 & 94.26 & 36.19 & 44.50 \\
SY3 & 90.38 & 36.62 & 47.79 \\
SY4 & 91.82 & 29.45 & 45.74 \\
PEDOT:PSS & 9.83 & 28.24 & 82.08 \\
\hline NiOx/SY1 & 79.51 & 23.69 & 50.40 \\
NiOx/SY2 & 86.68 & 32.03 & 48.81 \\
NiOx/SY3 $_{\mathbf{x}}$ & 84.07 & 30.38 & 54.34 \\
NiOx/SY4 $_{\mathbf{N}}$ & 88.15 & 26.56 & 50.08 \\
$\mathbf{N i O}_{\mathbf{x}}$ & 32.30 & 23.69 & 76.27 \\
\hline
\end{tabular}

a The HTM was annealed at $100^{\circ} \mathrm{C}$ for $10 \mathrm{~min}$. 


\section{XRD spectra}
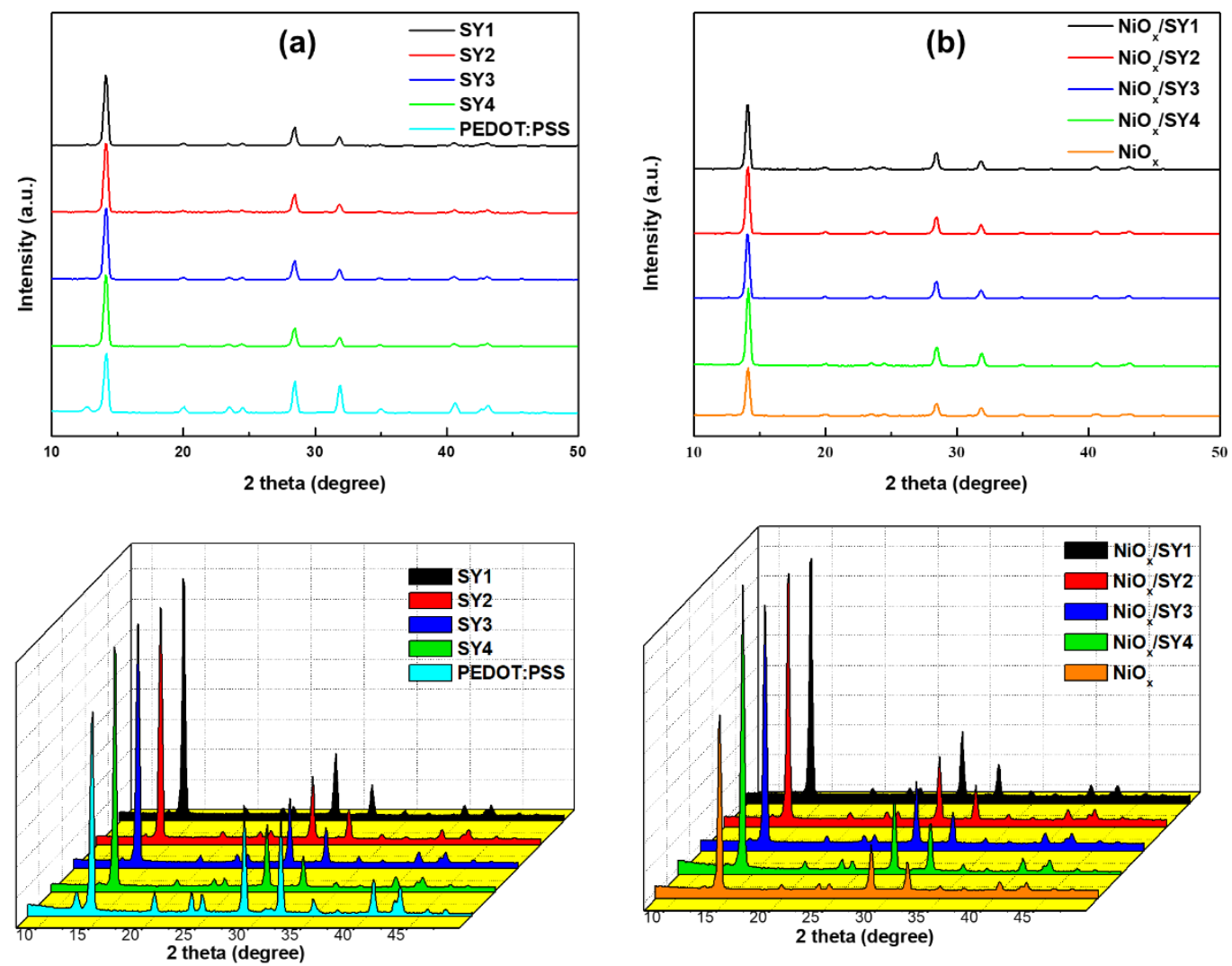

Figure S16. XRD spectra of perovskite films deposited on (a) SY1 SY4 and PEDOT:PSS as HTMs and (b) $\mathrm{NiO}_{x} / \mathbf{S Y} 1, \mathrm{NiO}_{x} / \mathbf{S Y 2}, \mathrm{NiO}_{x} / \mathbf{S Y 3}, \mathrm{NiO}_{x} / \mathbf{S Y 4}$, and bare $\mathrm{NiO}_{x}$ as HTMs.

\section{Cross-section SEM images}
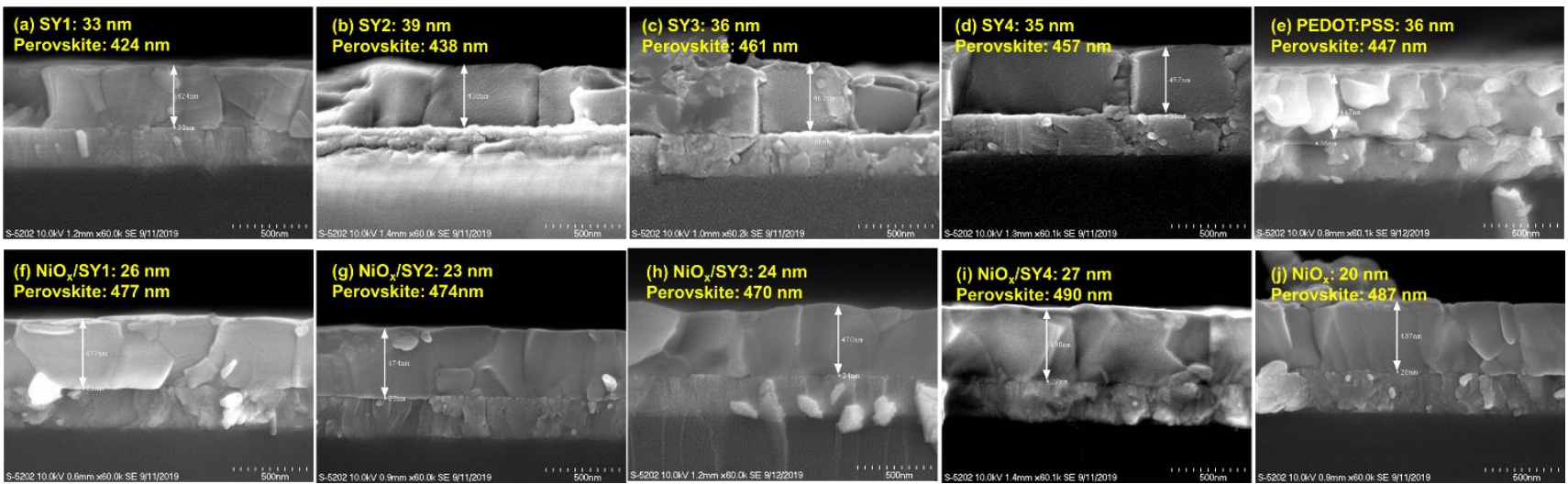
Figure S17. Cross-section SEM images of the perovskites on SY-HTMs/ITO.

11. PSCs device transparency

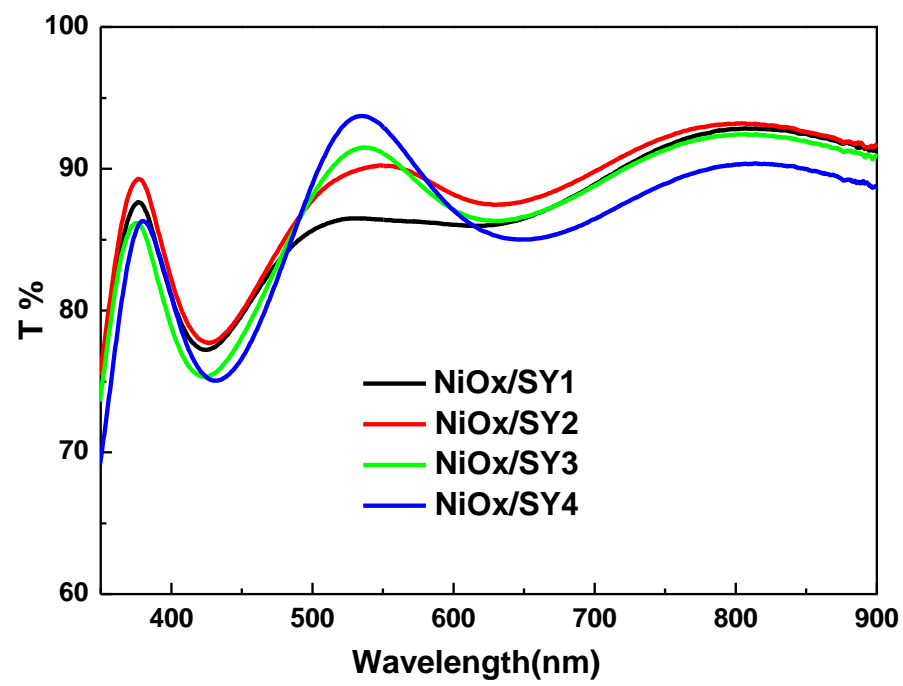

Figure S18. The UV-Vis transmittance of ITO/NiOx/SY1 4.

\section{EIS spectra}

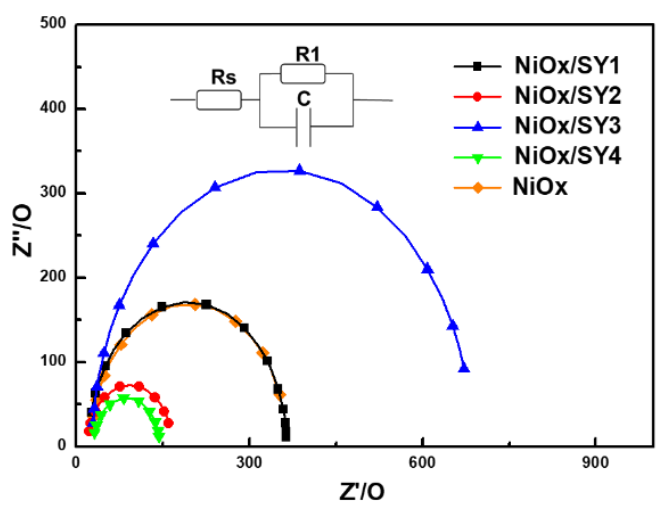

Humidity $=55 \mathrm{RH}$

\begin{tabular}{|c|c|c|}
\hline Device & Rs & R1 \\
\hline NiOx / SY1 & 21.4 & 343 \\
\hline NiOx / SY2 & 20.2 & 145.6 \\
\hline NiOx / SY3 & 28.6 & 656.7 \\
\hline NiOx / SY4 & 29.6 & 116.9 \\
\hline NiOx & 26.1 & 338.4 \\
\hline
\end{tabular}

Figure S19. Nyquist plots (i.e., minus imaginary part of the impedance $-Z^{\prime \prime}$ vs. the real part of the impedance $Z^{\prime}$ when sweeping the frequency) of the PSCs devices under illumination of $100 \mathrm{~mW} / \mathrm{cm}^{2}$. 


\section{PSC device stability}

Table S4. Variation of the performance of PSCs in device I and II under Ar condition.

\begin{tabular}{|c|c|c|c|c|}
\hline HTM & $J_{\mathrm{sc}}\left(\mathrm{mA} \cdot \mathrm{cm}^{-2}\right)$ & $V_{\mathrm{oc}}(\mathrm{V})$ & $\mathrm{FF}(\%)$ & PCE $(\%)$ \\
\hline SY1 (0 hr $)^{a}$ & 17.74 & 0.917 & 70.2 & 11.423 \\
\hline SY1 $(168 \mathrm{hr})^{\mathrm{a}}$ & 17.53 & 0.920 & 69.5 & 11.209 \\
\hline SY1 $(336 \text { hr })^{a}$ & 17.56 & 0.913 & 66.7 & 10.694 \\
\hline SY1 $(504 \text { hr })^{a}$ & 16.53 & 0.916 & 68.7 & 10.402 \\
\hline SY1 $(672 \mathrm{hr})^{\mathrm{a}}$ & 16.65 & 0.907 & 64.3 & 9.710 \\
\hline SY1 (840 hr) $)^{a}$ & 16.48 & 0.908 & 62.1 & 9.293 \\
\hline SY1 (1000 hr $)^{\mathrm{a}}$ & 18.51 & 0.906 & 50.6 & 8.486 \\
\hline SY2 $(0 \text { hr })^{\mathbf{a}}$ & 19.16 & 1.003 & 67.3 & 12.931 \\
\hline SY2 $(168 \mathrm{hr})^{\mathrm{a}}$ & 18.46 & 0.999 & 69.2 & 12.753 \\
\hline SY2 $(336 \text { hr })^{a}$ & 17.91 & 1.001 & 66.2 & 11.860 \\
\hline SY2 $(504 \text { hr })^{a}$ & 17.66 & 1.008 & 70.4 & 12.525 \\
\hline SY2 $(672 h r)^{a}$ & 18.26 & 0.988 & 64.7 & 11.676 \\
\hline SY2 $(840 \text { hr })^{a}$ & 16.89 & 0.981 & 65.2 & 10.802 \\
\hline SY2 $(1000 \mathrm{hr})^{\mathrm{a}}$ & 16.76 & 0.981 & 65.4 & 10.755 \\
\hline SY3 $(0 \text { hr })^{\mathrm{a}}$ & 17.25 & 0.950 & 67.5 & 11.066 \\
\hline SY3 (168 hr $)^{a}$ & 16.47 & 0.951 & 65.3 & 10.229 \\
\hline SY3 $(336 \mathrm{hr})^{\mathrm{a}}$ & 16.55 & 0.951 & 65.7 & 10.343 \\
\hline SY3 $(504 \text { hr })^{a}$ & 15.84 & 0.954 & 64.1 & 9.686 \\
\hline SY3 $(672 \text { hr })^{a}$ & 15.52 & 0.943 & 61.2 & 8.959 \\
\hline SY3 $(840 \text { hr })^{a}$ & 15.73 & 0.952 & 57.5 & 8.615 \\
\hline SY3 $(1000 \mathrm{hr})^{\mathrm{a}}$ & 14.98 & 0.958 & 55.2 & 7.923 \\
\hline SY4 $(0 \text { hr })^{\mathrm{a}}$ & 18.12 & 0.990 & 69.3 & 12.43 \\
\hline SY4 $(168 \mathrm{hr})^{\mathrm{a}}$ & 18.17 & 0.990 & 65.2 & 11.73 \\
\hline SY4 $(336 \mathrm{hr})^{\mathrm{a}}$ & 17.96 & 0.981 & 64.1 & 11.29 \\
\hline SY4 $(504 \mathrm{hr})^{\mathrm{a}}$ & 16.86 & 0.992 & 62.3 & 10.42 \\
\hline SY4 $(672 \mathrm{hr})^{\mathrm{a}}$ & 16.59 & 0.987 & 63.8 & 10.45 \\
\hline SY4 $(840 \text { hr })^{a}$ & 15.46 & 0.982 & 59.2 & 8.98 \\
\hline SY4 $(1000 \mathrm{hr})^{\mathrm{a}}$ & 15.73 & 0.982 & 58.8 & 9.08 \\
\hline PEDOT:PSS(0 hr $)^{\mathbf{a}}$ & 18.42 & 0.983 & 67.3 & 12.192 \\
\hline PEDOT:PSS(168 hr $)^{\mathrm{a}}$ & 18.62 & 0.975 & 64.6 & 11.731 \\
\hline PEDOT:PSS(336 hr $)^{\mathrm{a}}$ & 17.77 & 0.950 & 69.6 & 11.693 \\
\hline PEDOT:PSS(504 hr) ${ }^{\mathrm{a}}$ & 17.99 & 0.977 & 66.0 & 11.605 \\
\hline PEDOT:PSS(672 hr $)^{\mathrm{a}}$ & 16.91 & 0.967 & 56.7 & 9.274 \\
\hline PEDOT:PSS(840 hr $)^{\mathrm{a}}$ & 16.99 & 0.925 & 60.6 & 9.521 \\
\hline PEDOT:PSS $(1000 \mathrm{hr})^{\mathrm{a}}$ & 17.82 & 0.904 & 50.3 & 8.103 \\
\hline NiO $_{\mathbf{x}} /$ SY1 $(0 \text { hr })^{b}$ & 20.85 & 1.099 & 76.7 & 17.565 \\
\hline $\mathrm{NiO}_{\mathbf{x}} / \mathrm{SY} 1(168 \mathrm{hr})^{\mathrm{b}}$ & 21.31 & 1.095 & 74.6 & 17.406 \\
\hline $\mathrm{NiO}_{\mathbf{x}} / \mathrm{SY} 1(336 \mathrm{hr})^{\mathrm{b}}$ & 20.72 & 1.070 & 75.2 & 16.675 \\
\hline $\mathrm{NiO}_{\mathrm{x}} / \mathrm{SY} 1(504 \mathrm{hr})^{\mathrm{b}}$ & 20.88 & 1.083 & 74.2 & 16.762 \\
\hline $\mathrm{NiO}_{\mathrm{x}} / \mathrm{SY} 1(672 \mathrm{hr})^{\mathrm{b}}$ & 20.26 & 1.054 & 76.8 & 16.394 \\
\hline $\mathrm{NiO}_{\mathrm{x}} / \mathrm{SY} 1(840 \mathrm{hr})^{\mathrm{b}}$ & 20.11 & 1.054 & 75.9 & 16.099 \\
\hline $\mathrm{NiO}_{\mathbf{x}} / \mathrm{SY} 1(1000 \mathrm{hr})^{\mathrm{b}}$ & 20.00 & 1.055 & 77.0 & 16.245 \\
\hline $\mathrm{NiO}_{\mathrm{x}} / \mathrm{SY} 2(\mathbf{0} \mathrm{hr})^{\mathrm{b}}$ & 21.44 & 1.102 & 78.5 & 18.547 \\
\hline $\mathrm{NiO}_{\mathrm{x}} / \mathrm{SY} 2(168 \mathrm{hr})^{\mathrm{b}}$ & 21.47 & 1.079 & 76.1 & 17.269 \\
\hline $\mathrm{NiO}_{\mathrm{x}} / \mathrm{SY} 2(336 \mathrm{hr})^{\mathrm{b}}$ & 21.06 & 1.079 & 78.5 & 17.838 \\
\hline NiO $_{x} /$ SY2 $(504 \text { hr })^{b}$ & 21.08 & 1.067 & 76.2 & 17.139 \\
\hline $\mathrm{NiO}_{\mathrm{x}} / \mathrm{SY} 2(672 \mathrm{hr})^{\mathrm{b}}$ & 20.55 & 1.099 & 74.2 & 16.757 \\
\hline $\mathrm{NiO}_{\mathrm{x}} / \mathrm{SY} 2(840 \mathrm{hr})^{\mathrm{b}}$ & 20.63 & 1.085 & 73.9 & 16.541 \\
\hline $\mathrm{NiO}_{\mathbf{x}} / \mathrm{SY} 2(1000 \mathrm{hr})^{\mathrm{b}}$ & 20.47 & 1.077 & 75 & 16.534 \\
\hline NiOx$_{x} /$ SY3 $(0 \text { hr })^{b}$ & 20.14 & 1.060 & 71.7 & 15.305 \\
\hline \multirow[t]{2}{*}{$\mathrm{NiO}_{\mathrm{x}} / \mathrm{SY} 3(168 \mathrm{hr})^{\mathrm{b}}$} & 19.83 & 1.047 & 72.1 & 14.973 \\
\hline & & S-25 & & \\
\hline
\end{tabular}




\begin{tabular}{|c|c|c|c|c|}
\hline $\mathrm{NiO}_{\mathrm{x}} / \mathrm{SY} 3(336 \mathrm{hr})^{\mathrm{b}}$ & 19.82 & 1.044 & 73.3 & 15.163 \\
\hline NiO $_{x} /$ SY3 $(504 \text { hr })^{b}$ & 19.55 & 1.052 & 72.7 & 14.945 \\
\hline $\mathrm{NiO}_{\mathrm{x}} / \mathrm{SY} 3(672 \mathrm{hr})^{\mathrm{b}}$ & 19.21 & 1.066 & 71.8 & 14.693 \\
\hline $\mathrm{NiO}_{\mathbf{x}} / \mathrm{SY} 3(840 \mathrm{hr})^{\mathrm{b}}$ & 18.67 & 1.054 & 71.2 & 14.009 \\
\hline $\mathrm{NiO}_{\mathrm{x}} / \mathrm{SY} 3(1000 \mathrm{hr})^{\mathrm{b}}$ & 18.56 & 1.052 & 71.5 & 13.960 \\
\hline $\mathrm{NiO}_{\mathrm{x}} / \mathrm{SY} 4(\mathbf{0} \text { hr })^{\mathrm{b}}$ & 21.91 & 1.097 & 75.5 & 18.159 \\
\hline $\mathrm{NiO}_{\mathrm{x}} / \mathrm{SY} 4(168 \mathrm{hr})^{b}$ & 21.63 & 1.078 & 76.4 & 17.810 \\
\hline $\mathrm{NiO}_{\mathrm{x}} / \mathrm{SY} 4(336 \mathrm{hr})^{\mathrm{b}}$ & 21.48 & 1.066 & 76.5 & 17.508 \\
\hline $\mathrm{NiO}_{\mathrm{x}} / \mathrm{SY} 4(504 \mathrm{hr})^{\mathrm{b}}$ & 21.48 & 1.057 & 75.0 & 17.039 \\
\hline $\mathrm{NiO}_{\mathrm{x}} / \mathrm{SY} 4(672 \mathrm{hr})^{\mathrm{b}}$ & 21.23 & 1.068 & 75.2 & 17.051 \\
\hline $\mathrm{NiO}_{\mathrm{x}} / \mathrm{SY} 4(840 \mathrm{hr})^{\mathrm{b}}$ & 20.56 & 1.065 & 75.3 & 16.745 \\
\hline $\mathrm{NiO}_{\mathrm{x}} / \mathrm{SY} 4(1000 \mathrm{hr})^{\mathrm{b}}$ & 20.88 & 1.058 & 75.1 & 16.590 \\
\hline $\operatorname{NiO}_{x}(0 \text { hr })^{b}$ & 20.11 & 1.043 & 75.5 & 15.836 \\
\hline $\mathrm{NiO}_{x}(168 \mathrm{hr})^{b}$ & 19.95 & 1.054 & 74.5 & 15.663 \\
\hline $\mathrm{NiO}_{x}(336 \mathrm{hr})^{\mathrm{b}}$ & 20.00 & 1.052 & 74.2 & 15.606 \\
\hline $\mathrm{NiO}_{\mathrm{x}}(504 \mathrm{hr})^{\mathrm{b}}$ & 19.66 & 1.023 & 72.1 & 14.497 \\
\hline $\mathrm{NiO}_{x}(672 \mathrm{hr})^{b}$ & 19.80 & 1.019 & 72.7 & 14.662 \\
\hline $\mathrm{NiO}_{x}(840 \text { hr })^{b}$ & 19.62 & 1.016 & 73.2 & 14.585 \\
\hline $\mathrm{NiO}_{\mathbf{x}}(1000 \mathrm{hr})^{\mathrm{b}}$ & 20.59 & 1.016 & 69.6 & 14.565 \\
\hline
\end{tabular}

${ }^{a}$ Device type I: ITO/HTM/perovskite/PC ${ }_{61} \mathrm{BM} / \mathrm{BCP} / \mathrm{Ag} .{ }^{b}$ Device type II: ITO/NiO $/$ interfacial layer /perovskite/PC $61 \mathrm{BM} / \mathrm{BCP} / \mathrm{Ag}$.

(a)

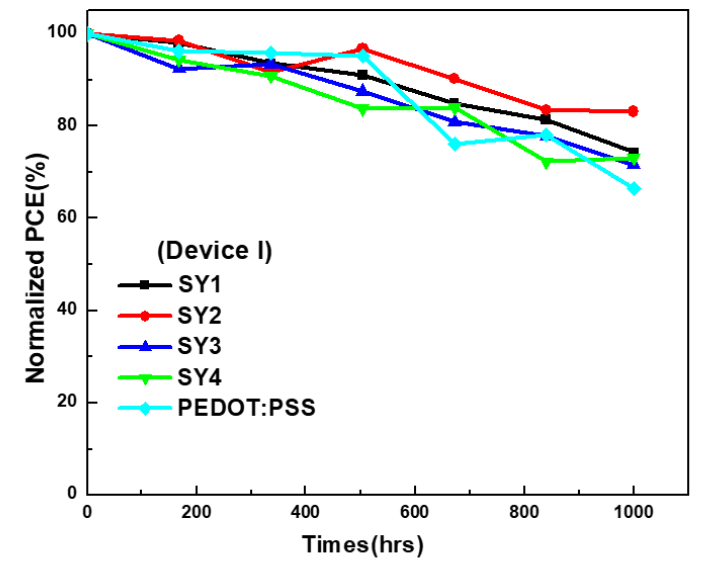

(b)

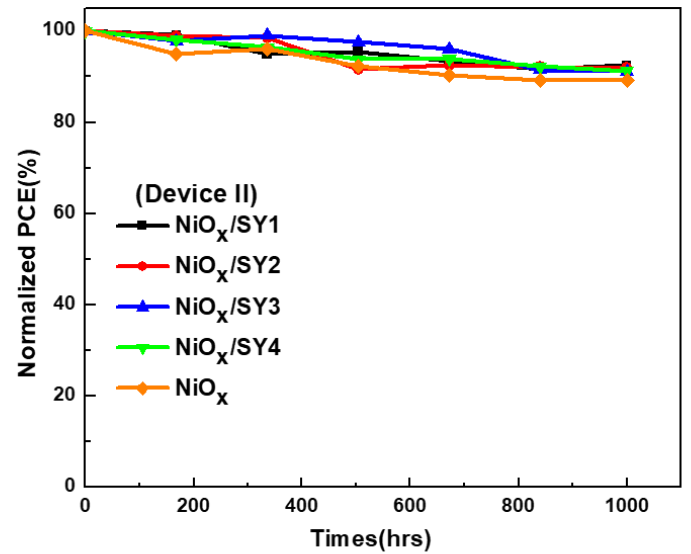

Figure S20. Stability tests of the PSCs under Ar condition incorporating (a) SY1, SY2, SY3, SY4, and PEDOT:PSS as HTMs (device I) and (b) $\mathrm{NiO}_{x} / \mathbf{S Y} \mathbf{1}, \mathrm{NiO}_{x} / \mathbf{S Y 2}, \mathrm{NiO}_{x} / \mathbf{S Y 3}, \mathrm{NiO}_{x} / \mathbf{S Y 4}$, and bare $\mathrm{NiO}_{x}$ as HTMs (device II). 


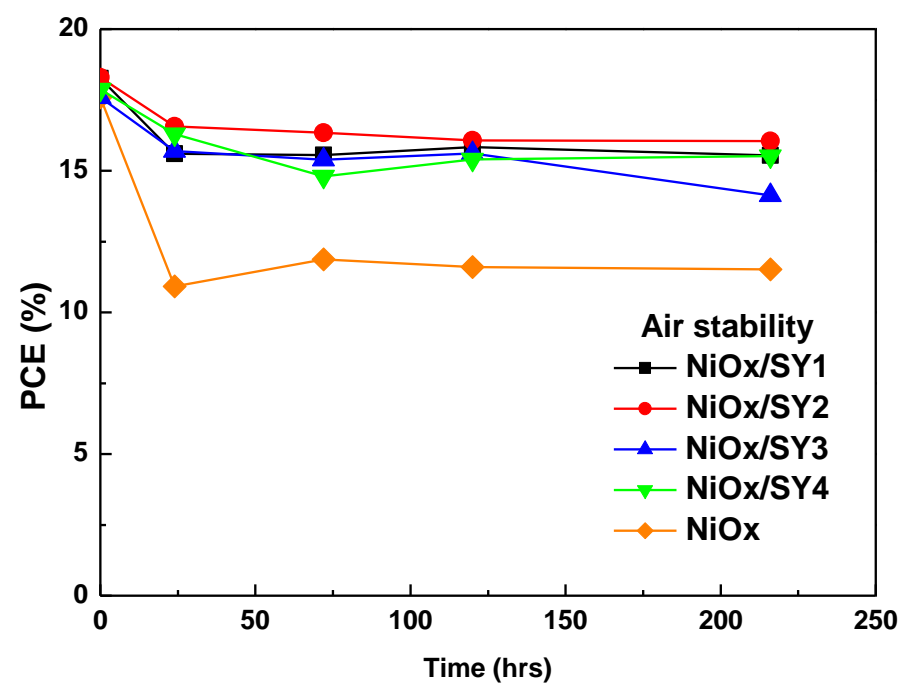

Figure S21. Stability tests of the PSCs under air condition $\left(25{ }^{\circ} \mathrm{C}\right.$, under air, $\left.40 \% \mathrm{RH}\right)$ incorporating

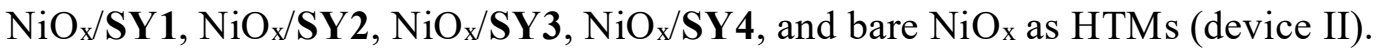

